\title{
Suzuki coupling of oxazoles
}

\author{
Emmanuel Ferrer, Matthew E. Popkin and Michael F. Greaney* \\ University of Edinburgh, School of Chemistry, Joseph Black Building, King’s Buildings, \\ West Mains Rd, Edinburgh EH9 3JJ, UK . \\ GlaxoSmithKline, Chemical Development, Old Powder Mills, Tonbridge, Kent, TN11 9AN, UK.
}

\section{Supporting Information}

\section{Part A: Experimental Procedures}

\section{General}

${ }^{1} \mathrm{H}-\mathrm{NMR}$ and ${ }^{13} \mathrm{C}$ NMR spectra were recorded on a Brüker dpx360 (360 MHz) and dpx250 (250 MHZ) instruments. Microwave reactions were carried out in a Smith Synthesizer Microwave (300 W). Melting point measurements were obtained from a Gallenkamp melting point apparatus and are uncorrected. Electrospray high resolution mass spectrometry was performed by the EPSRC National Mass Spectrometry Service Centre, Swansea, using a Finnigan MAT 900 XLT double focusing mass spectrometer. FAB HRMS was carried out by the University of Edinburgh School of Chemistry mass spectrometry service using a Kratos MS50 instrument. The data is recorded as the ionisation method followed by the calculated and measured masses. TLC was performed on Merck $60 \mathrm{~F}_{254}$ silica plates and visualised by UV light. The compounds were purified by wet flash chromatography using Merck Kieselgel 60 (particle size 35-70) silica under a positive pressure. 1,4 Dioxane was distilled over sodium and benzophenone prior use. Triethylamine and 2,6 lutidine were distilled over $\mathrm{CaH}_{2}$ prior to use. All other chemicals were purchased from a chemical supplier and used as received. 
- 2-Phenyloxazol-4-yl trifluoromethanesulfonate 2a

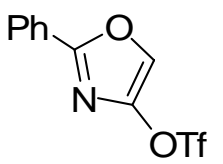

2-Phenyloxazol-4-yl trifluoromethanesulfonate was synthesised according to an established procedure: ${ }^{1} 2-$ Phenyl-4-oxazolone (3.00 g, $18.62 \mathrm{mmol}, 1$ equiv) was dissolved in DCM (75 mL, $0.25 \mathrm{M}$ ) and cooled to $-78^{\circ} \mathrm{C}$. To the solution was added $\mathrm{Et}_{3} \mathrm{~N}$ (5.3 mL, $37.24 \mathrm{mmol}$, 2 equiv), then very slowly $\mathrm{Tf}_{2} \mathrm{O}$ (4.8 mL, 27.93 mmol, 1.5 equiv). After warming to rt over $20 \mathrm{~min}$, the reaction was quenched with $\mathrm{H}_{2} \mathrm{O}$, extracted $3 \times$ into DCM. The organic layers were combined, washed with brine, dried over $\mathrm{MgSO}_{4}$, filtered and concentrated in vacuo. Purification by flash chromatography (silica, hexanes/ethyl acetate 1\%) gave the desired trifloyloxazole (4.90 g, 90\% yield) as a white solid (mp < $\left.25{ }^{\circ} \mathrm{C}\right) .{ }^{1} \mathbf{H}-\mathbf{N M R}\left(\mathrm{CDCl}_{3}, 250 \mathrm{MHz}\right) \delta$ 7.39-7.42 (3H, m), 7.65 (1H, s), 7.91$8.01(2 \mathrm{H}, \mathrm{m})$.

- 2-(4-Fluorophenyl)oxazol-4-yl trifluoromethanesulfonate $\mathbf{2 b}$

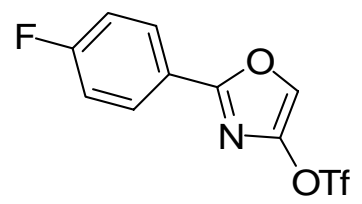

2-(4-Fluorophenyl)oxazol-4-yl trifluoromethanesulfonate was synthesised using Panek's method with minor modifications: ${ }^{1}$ In an argon-filled round bottom flask equipped with a reflux condenser were combined 4flurobenzamide (10.000 g, $70.4 \mathrm{mmol}, 1$ equiv) and chloroacetyl chloride (8.5 mL, $105.6 \mathrm{mmol}, 1.5$ equiv) and toluene $(100 \mathrm{~mL})$. The mixture was heated to $110{ }^{\circ} \mathrm{C}$ for $2 \mathrm{~h}$ until disappearance of the starting material by thin layer chromatography. Then, the mixture was allowed to cool down slowly and the chloroimide crystallised in the flask. Filtration, washing with hexane and drying in air yielded the chloroamide (11.708 g, $77 \%$ yield) as white needles; ${ }^{1} \mathrm{H}-\mathrm{NMR}\left(\mathrm{CDCl}_{3}, 250 \mathrm{MHz}\right) \delta 4.71$ (2H, s), 7.24-7.34 (2H, m), 7.93-7.34 (2H, m), $11.41(1 \mathrm{H}, \mathrm{s})$. ${ }^{13}$ C-NMR $\left(\mathrm{CDCl}_{3}, 63 \mathrm{MHz}\right) \delta 46.15\left(\mathrm{CH}_{2}\right), 115.94$ (quat, d, $J=21.9 \mathrm{~Hz}$ ), 129.34 (quat), 131.82 (quat, d, $J=9.4$ Hz), 165.25 (quat, d, $J=249.9 \mathrm{~Hz}$ ), 165.72 (quat), 168.83 (quat). The chloroamide product was then added to a mixture of sodium hydride (2.370 g, $59.24 \mathrm{mmol}, 1.1$ equiv, 60\% suspension in oil) and 1,4 dioxane (0.06 M) at $0{ }^{\circ} \mathrm{C}$. After stirring for $30 \mathrm{~min}$, the mixture was warmed to $\mathrm{rt}$ and stirred for $12 \mathrm{~h}$. The mixture was filtered through celite and concentrated in vacuo. Purification by flash chromatography (silica, hexane/ethyl acetate 4:6) gave the desired oxazolone (3.310 g, 34\% yield) as a white solid; ${ }^{1} \mathbf{H}-\mathbf{N M R}\left(\mathrm{CDCl}_{3}, 250 \mathrm{MHz}\right) \delta 4.69(2 \mathrm{H}, \mathrm{s})$, 7.11-7.21 (2H, m), 8.14-8.21 (2H, m). ${ }^{13} \mathrm{C}-\mathrm{NMR}\left(\mathrm{CDCl}_{3}, 63 \mathrm{MHz}\right) \delta 70.25\left(\mathrm{CH}_{2}\right), 116.91(\mathrm{CH}, \mathrm{d}, J=22.2 \mathrm{~Hz})$, 
122.20 (quat), 133.28 (CH, d, $J=9.8 \mathrm{~Hz}$ ), 165.30 (quat, d, $J=225.1 \mathrm{~Hz}$ ), 189.66 (quat). The pure oxazolone (3.240 g, 18.13 mmol, 1 equiv) was then dissolved in dry DCM and the solution cooled to $-78^{\circ} \mathrm{C}$. To the solution was added $\mathrm{Et}_{3} \mathrm{~N}$ (5.1 mL, $36.26 \mathrm{mmol}$, 2 equiv) and $\mathrm{Tf}_{2} \mathrm{O}$ (4.7 mL, $27.19 \mathrm{mmol}, 1.5$ equiv). After warming to rt over $1 \mathrm{~h}$, the reaction was quenched with $\mathrm{H}_{2} \mathrm{O}$ then extracted $3 \times$ into DCM. The organic layers were combined, dried over $\mathrm{MgSO}_{4}$, filtered and concentrated in vacuo. Purification by flash chromatography (silica, hexanes/ethyl acetate 9:1) gave the desired trifloyloxazole (5.027 g, 89\% yield) as a yellow solid (mp $<21{ }^{\circ} \mathrm{C}$ ); ${ }^{1}$ H-NMR $\left(\mathrm{CDCl}_{3}, 360 \mathrm{MHz}\right) \delta$ 7.13-7.18 (2H, m), 7.72 (1H, s), 7.98-8.03 (2H, m); ${ }^{13} \mathbf{C}-\mathbf{N M R}\left(\mathrm{CDCl}_{3}, 90 \mathrm{MHz}\right)$ $\delta 116.58(\mathrm{CH}, \mathrm{d}, J=16.3 \mathrm{~Hz}$ ), 118.97 (quat, d, $J=319.3 \mathrm{~Hz}), 122.82$ (quat, d, $J=3.2 \mathrm{~Hz}$ ), $126.71(\mathrm{CH}), 129.17$ (CH, d, J= 11.9 Hz), 146.32 (quat), 159.21 (quat), 165.04 (quat, d, $J=253.2 \mathrm{~Hz}$ ); HRMS (ES) calculated for $\mathrm{C}_{10} \mathrm{H}_{5} \mathrm{NO}_{4} \mathrm{SF}_{4} ; 311.9948$, found 311.9946 .

- 2-(4-Methoxyphenyl)oxazol-4-yl trifluoromethanesulfonate 2c

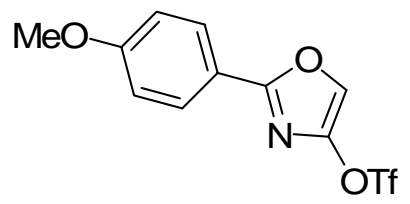

2-(4-methoxyphenyl)oxazol-4-yl trifluoromethanesulfonate was synthesised according to the established procedure and gave identical spectral data to that previously reported. ${ }^{2}{ }^{1} \mathbf{H}-\mathbf{N M R}\left(\mathrm{CDCl}_{3}, 250 \mathrm{MHz}\right) \delta 3.81(3 \mathrm{H}$, s), 6.87-6.93 (2H, m), 7.61 (1H, s), 7.84-7.90 (2H, m).

- 4-Phenyloxazol-2-yl nonafluorobutanesulfonate 6

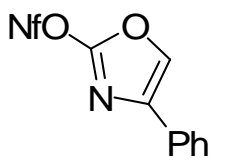

4-Phenyl-2-oxazolone (100 mg, $0.62 \mathrm{mmol}, 1$ equiv) was dissolved in DCM (8 mL, 0.25M) and cooled to -78 ${ }^{\circ} \mathrm{C}$. To the solution was added 2,6-Lutidine $\left(0.14 \mathrm{~mL}, 1.24 \mathrm{mmol}, 2\right.$ equiv) followed by dropwise addition of $\mathrm{Nf}_{2} \mathrm{O}$ ( $0.29 \mathrm{~mL}, 0.93 \mathrm{mmol}, 1.5$ equiv). After warming to rt over $20 \mathrm{~min}$, the reaction was quenched with $\mathrm{H}_{2} \mathrm{O}$, extracted $3 \times$ into DCM. The organic layers were combined, washed with brine, dried over $\mathrm{MgSO}_{4}$, filtered and concentrated in vacuo. Purification by flash chromatography (silica, hexanes/DCM 7:3) gave the desired nonaflate (230 mg, 84\% yield) as a pale yellow oil; ${ }^{1} \mathbf{H}-\mathrm{NMR}\left(\mathrm{CDCl}_{3}, 360 \mathrm{MHz}\right) \delta$ 7.37-7.45 (3H, m), 7.68-7.71 (2H, m), $\left.7.79(1 \mathrm{H}, \mathrm{s}) ;{ }^{13} \mathrm{C}-\mathrm{NMR}\left(\mathrm{CDCl}_{3}, 90 \mathrm{MHz}\right) \delta 125.44(\mathrm{CH}), 128.87 \mathrm{CH}\right), 129.09(\mathrm{CH}), 129.26$ (quat), 
132.70 (CH), 141.68 (quat), 150.11 (quat); ${ }^{19}$ F-NMR ( $\left.\mathrm{CDCl}_{3}, 250 \mathrm{MHz}\right):-127.0$ (2F, m), -121.9 (2F, m), $108.0(2 \mathrm{~F}, \mathrm{t}, J=13.4 \mathrm{~Hz}),-81.8(3 \mathrm{~F}, \mathrm{t}, J=9.6 \mathrm{~Hz})$.

General procedure for the synthesis of 2,4 diaryl-oxazoles through Suzuki coupling of 2-aryl-oxazol-4-yl trifluoromethanesulfonates with aryl boronic acids: The following procedure for the preparation of $3 \mathbf{a}$ is representative.

- 2-Phenyl-4-p-tolyloxazole 3a

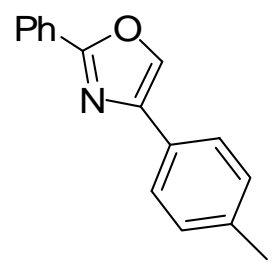

A microwave vial was charged with 2-phenyloxazol-4-yl trifluoromethanesulfonate (100 mg, 0.34 mmol, 1 equiv), 4-tolylboronic acid (52 mg, $0.37 \mathrm{mmol}, 1.1$ equiv), $\mathrm{PdCl}_{2}\left(\mathrm{PPh}_{3}\right)_{2}$ (5 mol \%), sodium carbonate (0.53 mL 2M, 1.06 mmol, 3 equiv) and 1,4 dioxane (5 mL). The vial was sealed and stirred until complete dissolution of the boronic acid occurred. The mixture was then irradiated for 20 minutes at a pre-selected temperature of $150{ }^{\circ} \mathrm{C}$ in a Smith synthesiser. The vial was then cooled with air jet cooling and the crude reaction mixture filtered through a pad of celite ${ }^{\circledR}$ and washed thoroughly with acetone. The organic layers were concentrated in vacuo and the residue was purified by flash chromatography (silica, hexanes/DCM 6:4) to give the coupled product 3 (75 mg, 94\% yield) as a white solid; $\mathrm{mp}=110-11{ }^{\circ} \mathrm{C}$; ${ }^{1} \mathbf{H}-\mathbf{N M R}\left(\mathrm{CDCl}_{3}, 250 \mathrm{MHz}\right): \delta 2.44$ (3H, s), 7.31-7.35 (2H, m), 7.46-7.49 (3H, m), 7.75-7.77 (2H, m), 7.98 (1H, s), 8.18-8.16 (2H, m); ${ }^{13} \mathbf{C}-\mathbf{N M R}\left(\mathrm{CDCl}_{3}, 63 \mathrm{MHz}\right) \delta$ $21.31\left(\mathrm{CH}_{3}\right), 125.50(\mathrm{CH}), 126.46(\mathrm{CH}), 127.54$ (quat), 128.26 (quat), $128.71(\mathrm{CH}), 129.40(\mathrm{CH}), 130.29(\mathrm{CH})$, 132.98 (CH), 137.91 (quat), 142.00 (quat), 161.78 (quat); HRMS (ES) calculated for $\mathrm{C}_{16} \mathrm{H}_{13} \mathrm{NO} 236.1070$; found 236.1069.

- 4-(3-Nitrophenyl)-2-phenyloxazole 3b

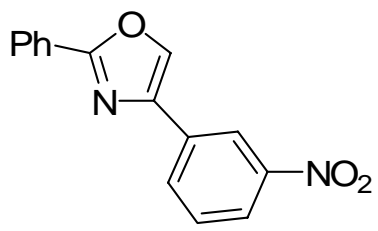

Prepared according to the general procedure. Purification by flash chromatography (silica, hexanes/DCM 5:5) gave the coupled product (83 mg, 92\% yield) as a white solid; mp = 161-163 ${ }^{\circ} \mathrm{C} ;{ }^{1} \mathbf{H}-\mathbf{N M R}\left(\mathrm{CDCl}_{3}, 250 \mathrm{MHz}\right): \delta$ 
7.57-7.60 (3H, m), 7.69 (1H, dd, $J=8.0 \mathrm{~Hz}, J=8.0 \mathrm{~Hz}), 8.21-8.25(5 \mathrm{H}, \mathrm{m}), 8.75(1 \mathrm{H}, \mathrm{s}) ;{ }^{13} \mathrm{C}-\mathrm{NMR}\left(\mathrm{CDCl}_{3}, 63\right.$ MHz) $\delta 120.32(\mathrm{CH}), 122.54(\mathrm{CH}), 126.47(\mathrm{CH}), 126.84$ (quat), $128.74(\mathrm{CH}), 129.60(\mathrm{CH}), 130.69(\mathrm{CH})$, $131.20(\mathrm{CH}), 132.83$ (quat), 134.43 (CH), 139.91 (quat), 148.53 (quat), 162.29 (quat); HRMS (ES) calculated for $\mathrm{C}_{15} \mathrm{H}_{10} \mathrm{~N}_{2} \mathrm{O}_{2}$ 267.0764; found 267.0761.

- 2-Phenyl-4-(4-(trifluoromethyl)phenyl)oxazole 3c

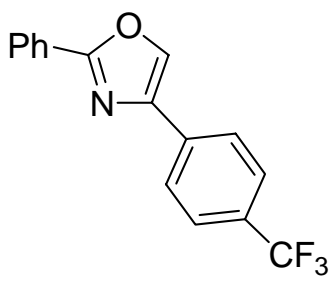

Prepared according to the general procedure. Purification by flash chromatography (silica, hexanes/DCM 6:4) gave the coupled product (86 mg, 87\% yield) as a white solid; $\mathrm{mp}=144-145{ }^{\circ} \mathrm{C}$;. ${ }^{1} \mathbf{H}-\mathbf{N M R}\left(\mathrm{CDCl}_{3}, 250 \mathrm{MHz}\right)$ :

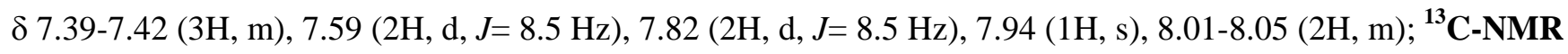
$\left(\mathrm{CDCl}_{3}, 90 \mathrm{MHz}\right) \delta 125.47$ (quat), $125.66(\mathrm{CH}), 125.73(\mathrm{CH}), 126.28$ (quat), $127.14(\mathrm{CH}), 128.3$ (quat, q, J= $277.4 \mathrm{~Hz}$ ), 128.69 (CH), 128.80 (quat), 130.66 (CH), 134.42 (CH), 140.77 (quat), 162.26 (quat); HRMS (ES) calculated for $\mathrm{C}_{16} \mathrm{H}_{10} \mathrm{NOF}_{3}$ 290.0787; found 290.0788.

- 2-Phenyl-4-o-tolyloxazole 3d

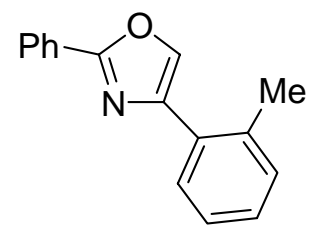

Prepared according to the general procedure. Purification by flash chromatography (silica, hexanes/DCM 5:5) gave the coupled product (73 mg, 91\% yield) as a white solid; mp $=67-68{ }^{\circ} \mathrm{C} ;{ }^{1} \mathbf{H}-\mathbf{N M R}\left(\mathrm{CDCl}_{3}, 250 \mathrm{MHz}\right): \delta$ 2.50 (3H, s), 7.22-7.30 (3H, m), 7.46-7.49 (3H, m), 7.81 (1H, s), 7.93 (1H, d, J= 6.5Hz), 8.11-8.15 (2H, m); ${ }^{13} \mathrm{C}-$ NMR $\left(\mathrm{CDCl}_{3}, 63 \mathrm{MHz}\right) \delta 22.72\left(\mathrm{CH}_{3}\right), 126.05(\mathrm{CH}), 126.47(\mathrm{CH}), 127.47$ (quat), $127.93(\mathrm{CH}), 128.66(\mathrm{CH})$, $128.71(\mathrm{CH}), 130.31$ (quat), $130.38(\mathrm{CH}), 131.76(\mathrm{CH}), 135.20(\mathrm{CH}), 135.54$ (quat), 140.97 (quat), 160.88 (quat); HRMS (ES) calculated for $\mathrm{C}_{16} \mathrm{H}_{13} \mathrm{NO} 236.1070$; found 236.1071. 
- 2-(4-Fluorophenyl)-4-p-tolyloxazole 3e

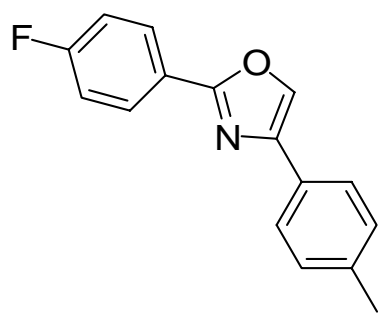

Prepared according to the general procedure. Purification by flash chromatography (silica, hexanes/DCM 5:5) gave the coupled product (61 mg, 75\% yield) as a white solid; mp $=140-141{ }^{\circ} \mathrm{C} ;{ }^{1} \mathbf{H}-\mathbf{N M R}\left(\mathrm{CDCl}_{3}, 250 \mathrm{MHz}\right)$ :

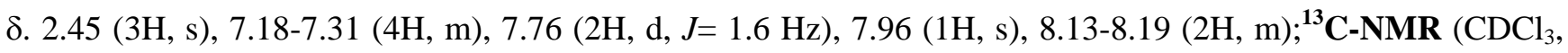
$63 \mathrm{MHz}$ ) $\delta .21 .29\left(\mathrm{CH}_{3}\right), 115.87$ (CH, d, $\left.J=22 \mathrm{~Hz}\right), 123.89$ (quat), 125.48 (CH), 128.15 (quat), 128.57 (CH, d, $J=$ 8.6 Hz, 129.41 (CH), 132.97 (CH), 137.98 (quat), 142.03 (quat), 160.95 (quat), 164.02 (quat, d, $J=249.3$ Hz); HRMS (FAB) calculated for $\mathrm{C}_{16} \mathrm{H}_{12} \mathrm{ONF} 254.09812$; found 254.09861.

- 4-(4-Methoxyphenyl)-2-phenyloxazole $3 \mathbf{f}^{3}$

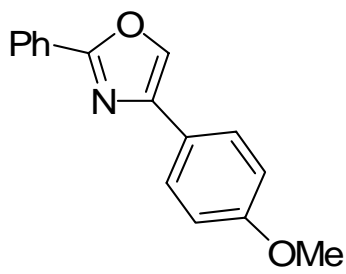

Prepared according to the general procedure. Purification by flash chromatography (silica, hexanes/DCM 6:4) gave the coupled product (76 mg, 89\% yield) as a white solid. This compound showed identical spectral data to that previously reported; ${ }^{3} \mathrm{mp}=105-106{ }^{\circ} \mathrm{C} ;{ }^{1} \mathbf{H}-\mathrm{NMR}\left(\mathrm{CDCl}_{3}, 250 \mathrm{MHz}\right): \delta 4.04$ (3H, s), 7.16 (2H, d, J=8.9 Hz), 7.65-7.67 (3H, m), 7.94 (2H, d, J= 8.9 Hz), 8.06 (1H, s), 8.10-8.14 (2H, m); ${ }^{13}$ C-NMR $\left(\mathrm{CDCl}_{3}, 63 \mathrm{MHz}\right) \delta 55.28$ $\left(\mathrm{CH}_{3}\right), 114.12(\mathrm{CH}), 123.80$ (quat), $126.43(\mathrm{CH}), 126.90(\mathrm{CH}), 127.54$ (quat), $128.70(\mathrm{CH}), 130.26(\mathrm{CH}), 132.37$ (CH), 141.74 (quat), 159.51 (quat), 161.73 (quat). 
- 2-(4-Methoxyphenyl)-4-p-tolyloxazole 3g

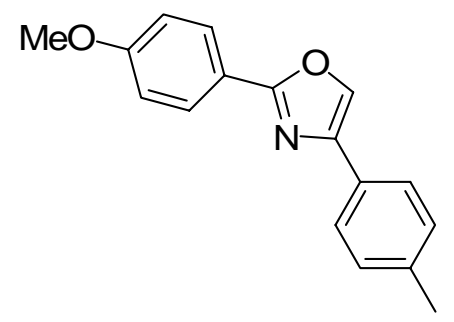

Prepared according to the general procedure. Purification by flash chromatography (silica, hexanes/DCM 2:8) gave the coupled product (68 mg, 82\% yield) as a white solid; mp $=130-131{ }^{\circ} \mathrm{C} ;{ }^{1} \mathbf{H}-\mathbf{N M R}\left(\mathrm{CDCl}_{3}, 360 \mathrm{MHz}\right): \delta$ 2.44 (3H, s), 3.90 (3H, s), 7.03 (2H, d, $J=8.5 \mathrm{~Hz}), 7.29$ (2H, d, $J=8.5 \mathrm{~Hz}), 7.76$ (2H, d, J= $8.1 \mathrm{~Hz}), 7.92$ (1H, s), $8.11(2 \mathrm{H}, \mathrm{d}, J=11.8 \mathrm{~Hz}) ;{ }^{13} \mathrm{C}-\mathrm{NMR}\left(\mathrm{CDCl}_{3}, 90 \mathrm{MHz}\right) \delta 21.27\left(\mathrm{CH}_{3}\right), 55.31\left(\mathrm{CH}_{3}\right), 114.08(\mathrm{CH}), 120.38$ (quat), $125.45(\mathrm{CH}), 128.10 \mathrm{CH}), 128.41$ (quat), $129.34(\mathrm{CH}), 132.44(\mathrm{CH}), 137.74$ (quat), 141.76 (quat), 161.27 (quat), 161.83 (quat); HRMS (ES) calculated for $\mathrm{C}_{17} \mathrm{H}_{15} \mathrm{NO}_{2}$ 266.1176; found 266.1176.

- 4-(Furan-3-yl)-2-phenyloxazole $\mathbf{3 h}$

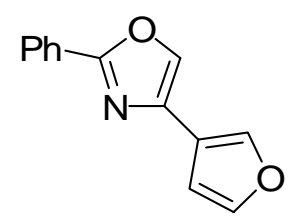

Prepared according to the general procedure. Purification by flash chromatography (silica, hexanes/DCM 5:5) gave the coupled product (57 mg, 79\% yield) as a yellow solid; mp $=81-82{ }^{\circ} \mathrm{C} ;{ }^{1} \mathbf{H}-\mathbf{N M R}\left(\mathrm{CDCl}_{3}, 360 \mathrm{MHz}\right): \delta$ $6.65(1 \mathrm{H}, \mathrm{s}), 7.45-7.48$ (4H, m), $7.76(1 \mathrm{H}, \mathrm{s}), 7.90$ (1H, s), 8.06-8.09 (2H, m); ${ }^{13} \mathbf{C}-\mathbf{N M R}\left(\mathrm{CDCl}_{3}, 90 \mathrm{MHz}\right) \delta$ 108.37 (CH), 117.20 (quat), $126.47(\mathrm{CH}), 127.27$ (quat), $128.73(\mathrm{CH}), 130.44(\mathrm{CH}), 133.08(\mathrm{CH}), 134.98$ (quat), $139.92(\mathrm{CH}), 143.60(\mathrm{CH}), 161.90$ (quat); HRMS (ES) calculated for $\mathrm{C}_{13} \mathrm{H}_{9} \mathrm{NO}_{2}$ 212.0706; found 212.0706.

- 3-(2-Phenyloxazol-4-yl)pyridine 3i

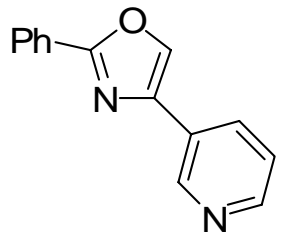


Prepared according to the general procedure. Purification by flash chromatography (silica, hexanes/DCM 5:5) gave the coupled product (55 mg, 73\% yield) as a yellow solid; mp $=92-93{ }^{\circ} \mathrm{C} ;{ }^{1} \mathbf{H}-\mathbf{N M R}\left(\mathrm{CDCl}_{3}, 360 \mathrm{MHz}\right): \delta$ 7.38(1H, m), 7.36-7.39 (3H, m), $8.04(1 \mathrm{H}, \mathrm{s}), 8.10-8.16$ (3H, m), $8.57(1 \mathrm{H}, \mathrm{m}), 9.05(1 \mathrm{H}, \mathrm{m}) ;{ }^{13} \mathrm{C}-\mathrm{NMR}\left(\mathrm{CDCl}_{3}\right.$, $90 \mathrm{MHz}) \delta 124.80(\mathrm{CH}), 127.61(\mathrm{CH}), 128.13$ (quat), 128.44 (quat), $129.88(\mathrm{CH}), 131.76(\mathrm{CH}), 134.25(\mathrm{CH})$, $135.11(\mathrm{CH}), 140.10$ (quat), $147.80(\mathrm{CH}), 149.83(\mathrm{CH}), 163.47$ (quat); HRMS (ES) calculated for $\mathrm{C}_{14} \mathrm{H}_{10} \mathrm{~N}_{2} \mathrm{O}$ 223.0866; found 223.0867.

- 3-(2-(4-Methoxyphenyl)oxazol-4-yl)pyridine 3j

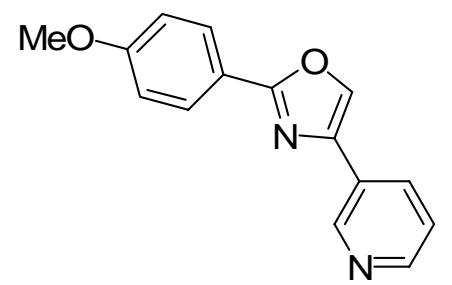

Prepared according to the general procedure. Purification by flash chromatography (silica, EtOAc) gave the coupled product (70 mg, 89\% yield) as a white solid; mp = 120-122 ${ }^{\circ} \mathrm{C}$; ${ }^{1} \mathbf{H}-\mathrm{NMR}$ (DMSO, $250 \mathrm{MHz}$ ): $\delta 3.79$ (3H, $\left.\mathrm{CH}_{3}\right), 7.06(2 \mathrm{H}, \mathrm{d}, J=8.8 \mathrm{~Hz}), 7.46(1 \mathrm{H}, \mathrm{m}), 7.58(2 \mathrm{H}, \mathrm{d}, J=4.1 \mathrm{~Hz}), 7.95(1 \mathrm{H}, \mathrm{d}, J=8.8 \mathrm{~Hz}) 8.17(1 \mathrm{H}, \mathrm{m})$, 8.73 (1H, s), 9.03 (1H, bs); ${ }^{13}$ C-NMR (DMSO, $\left.63 \mathrm{MHz}\right) \delta 55.35\left(\mathrm{CH}_{3}\right), 114.55(\mathrm{CH}), 119.07$ (quat), 12.88 (CH), 126.82 (quat), $127.90(\mathrm{CH}), 132.51(\mathrm{CH}), 135.74(\mathrm{CH}), 138.05$ (quat), $146.32(\mathrm{CH}), 148.78(\mathrm{CH}), 161.27$ (quat), 161.44 (quat); HRMS (ES) calculated for $\mathrm{C}_{15} \mathrm{H}_{12} \mathrm{~N}_{2} \mathrm{O}_{2}$ 253.0972; found 253.0972.

- 4-(3-Fluorophenyl)-2-phenyloxazole 3k

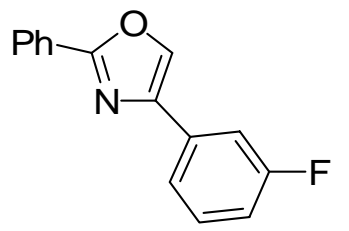

Prepared according to the general procedure. Purification by flash chromatography (silica, hexanes/DCM 6:4) gave the coupled product (74 mg, 91\% yield) as a white solid; mp= 71-72 ${ }^{\circ} \mathrm{C}$; ${ }^{1} \mathbf{H}-\mathbf{N M R}\left(\mathrm{CDCl}_{3}, 250 \mathrm{MHz}\right): \delta$ $6.92(1 \mathrm{H}, \mathrm{m}), 7.28-7.40$ (6H, m), $7.87(1 \mathrm{H}, \mathrm{s}), 8.00-8.04(2 \mathrm{H}, \mathrm{m}) ;{ }^{13} \mathrm{C}-\mathrm{NMR}\left(\mathrm{CDCl}_{3}, 63 \mathrm{MHz}\right) \delta 112.60(\mathrm{CH}, \mathrm{d}$, $J=23 \mathrm{~Hz}), 114.87(\mathrm{CH}, \mathrm{d}, J=21 \mathrm{~Hz}), 121.16(\mathrm{CH}), 126.51(\mathrm{CH}), 127.24$ (quat), $128.76(\mathrm{CH}), 130.19(\mathrm{CH})$, 130.53 (CH), 133.90 (quat), 141.02 (quat), 163.35 (quat, d, $J=245 \mathrm{~Hz}$ ), 162.03 (quat); HRMS (ES) calculated for $\mathrm{C}_{15} \mathrm{H}_{10} \mathrm{NOF} 240.0819$; found 240.0818 . 
- 4-(3-Fluorophenyl)-2-(4-methoxyphenyl)oxazole 31

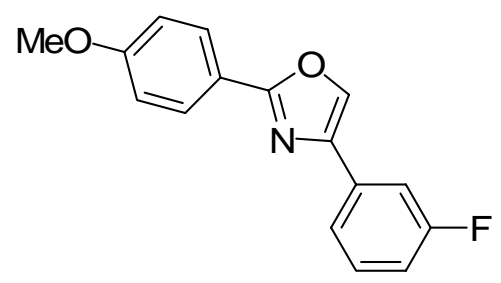

Prepared according to the general procedure. Purification by flash chromatography (silica, hexanes/DCM 2:8) gave the coupled product (71 mg, 85\% yield) as a white solid; $\mathrm{mp}=87-88{ }^{\circ} \mathrm{C} ;{ }^{1} \mathbf{H}-\mathbf{N M R}\left(\mathrm{CDCl}_{3}, 250 \mathrm{MHz}\right): \delta$ 3.77 (3H, s), 6.89 (3H, d, J= 7.4 Hz), 7.28 (1H, m), 7.46-7.49 (2H, m), 7.82 (1H, s), 7.95 (2H, d, J= $7.4 \mathrm{~Hz})$; ${ }^{13} \mathrm{C}-$ NMR $\left(\mathrm{CDCl}_{3}, 63 \mathrm{MHz}\right) \delta 55.35\left(\mathrm{CH}_{3}\right), 112.54(\mathrm{CH}, \mathrm{d}, J=22.9 \mathrm{~Hz}), 114.16(\mathrm{CH}), 114.75(\mathrm{CH}, \mathrm{d}, J=21.1 \mathrm{~Hz})$, 121.10 (quat), 121.14 (CH), 128.20 (CH), 130.21 (CH, d, $J=0.2 \mathrm{~Hz}$ ), 133.37 (CH), 133.48 (quat), 140.70 (quat), 161.49 (quat), 162.11 (quat), 163.13 (quat, d, $J=245.3 \mathrm{~Hz}$ ); HRMS (ES) calculated for $\mathrm{C}_{16} \mathrm{H}_{12} \mathrm{NO}_{2} \mathrm{~F} 270.0925$; found 270.0928 .

General procedure for the synthesis of 2,4 diaryl-oxazoles through Suzuki coupling of 2-chloro-4-phenyloxazole with aryl boronic acids. The procedure for compound 9a is representative:

- 4-Phenyl-2-o-tolyloxazole 9a

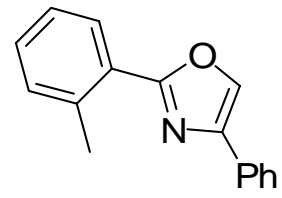

A microwave vial was charged with 2-chloro-4-phenyl oxazole (100 mg, $0.55 \mathrm{mmol}, 1$ equiv), o-tolylboronic acid (83 mg, $0.61 \mathrm{mmol}, 1.1$ equiv), $\mathrm{PdCl}_{2}\left(\mathrm{PPh}_{3}\right)_{2}$ (5 mol \%), sodium carbonate (0.82 mL $2 \mathrm{M}, 1.65 \mathrm{mmol}, 3$ equiv) and 1,4 dioxane (5 mL). The vial was sealed and stirred until complete dissolution of the boronic acid occurred. The mixture was then irradiated for 20 minutes at a pre-selected temperature of $150{ }^{\circ} \mathrm{C}$ in a Smith synthesiser. The vial was then cooled with air jet cooling and the crude reaction mixture filtered through a pad of celite $^{\circledR}$ and washed thoroughly with acetone. The organic layers were concentrated in vacuo and the residue was purified by flash chromatography (silica, hexanes/DCM 7:3) to give the coupling product as a yellow oil (104 mg, 80\% yield); ${ }^{1} \mathbf{H}-\mathbf{N M R}\left(\mathrm{CDCl}_{3}, 250 \mathrm{MHz}\right) \delta 2.54$ (3H, s), 7.07-7.13 (3H, m), 7.17-7.23 (3H, m), 7.59-7.63 (2H, m), $7.75(1 \mathrm{H}, \mathrm{s}), 7.83(1 \mathrm{H}, \mathrm{m}) ;{ }^{13} \mathrm{C}-\mathrm{NMR}\left(\mathrm{CDCl}_{3}, 63 \mathrm{MHz}\right) \delta 21.99\left(\mathrm{CH}_{3}\right), 125.57(\mathrm{CH}), 125.89(\mathrm{CH})$, 
126.42 (quat), $127.99(\mathrm{CH}), 128.69(\mathrm{CH}), 128.84(\mathrm{CH}), 129.97(\mathrm{CH}), 131.22$ (quat), $131.56(\mathrm{CH}), 132.95$ (CH), 137.64 (quat), 141.55 (quat), 162.24 (quat); HRMS (ES) calculated for $\mathrm{C}_{16} \mathrm{H}_{13} \mathrm{NO} 236.1070$; found 236.1070.

- 2-(3-Fluorophenyl)-4-phenyloxazole $\mathbf{9 b}$

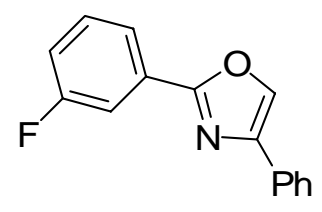

Prepared according to the general procedure. Purification by flash chromatography (silica, hexanes/DCM 6:4) gave the coupled product (106 mg, 80\% yield) as a white solid; mp $=70-72{ }^{\circ} \mathrm{C} ;{ }^{1} \mathbf{H}-\mathbf{N M R}\left(\mathrm{CDCl}_{3}, 250 \mathrm{MHz}\right) \delta$ 7.41 (1H, m), 7.63-7.70 (4H, m), 8.06-8.20 (5H, m); ${ }^{13} \mathrm{C}-\mathrm{NMR}\left(\mathrm{CDCl}_{3}, 63 \mathrm{MHz}\right) \delta 113.42$ (CH, d, J= 23.8 Hz), 117.28 (CH, d, $J=21.2 \mathrm{~Hz}$ ), $122.13(\mathrm{CH}, \mathrm{d}, J=3.0 \mathrm{~Hz}$ ), 125.31 (quat), 125.57 (CH), $128.19(\mathrm{CH}), 128.76(\mathrm{CH})$, 130.47 (CH, d, $J=8.1 \mathrm{~Hz}$ ), 130.80 (quat), 133.69 (CH), 135.35 (quat), 142.14 (quat), 163.83 (quat, d, $J=244.7$ $\mathrm{Hz}$ ); HRMS (ES) calculated for $\mathrm{C}_{15} \mathrm{H}_{10} \mathrm{NOF} 240.0819$; found 240.0819 .

- 4-Phenyl-2-p-tolyloxazole 9c

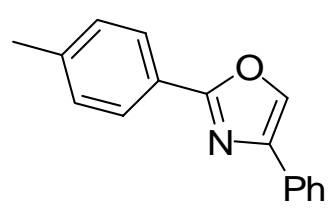

Prepared according to the general procedure. Purification by flash chromatography (silica, hexanes/DCM 6:4) gave the coupled product (113 mg, 88\% yield) as a white solid with identical spectral data to that previously published; ${ }^{4}{ }^{1}$ H-NMR $\left(\mathrm{CDCl}_{3}, 250 \mathrm{MHz}\right) \delta 2.41$ (3H, s), 7.25-7.44 (5H, m), 7.82-8.02 (4H, m), 7.94 (1H, s).

- 3-(4-Phenyloxazol-2-yl)pyridine 9d

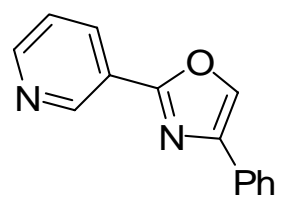


Prepared according to the general procedure. Purification by flash chromatography (silica, hexanes/Et ${ }_{2} \mathrm{O} 4: 6$ ) gave the coupled product (111 mg, 91\% yield) as a white solid with identical spectral data to that previously published; ${ }^{1}$ H-NMR $\left(\mathrm{CDCl}_{3}, 250 \mathrm{MHz}\right) \delta$ 7.27-7.46 (4H, m), 7.79-7.82 (2H, m), 7.99 (1H, s), 8.34-8.37 (1H, m), 8.67 (1H, d, $J=4 \mathrm{~Hz}), 9.33(1 \mathrm{H}, \mathrm{bs})$.

- 2-(Furan-3-yl)-4-phenyloxazole 9e

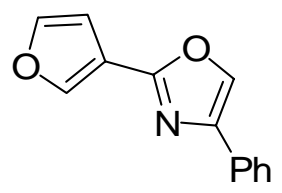

Prepared according to the general procedure. Purification by flash chromatography (silica, hexanes/DCM 5:5) gave the coupled product (95 mg, 82\%) as a white solid; mp $=78-79{ }^{\circ} \mathrm{C} ;{ }^{1} \mathbf{H}-\mathbf{N M R}\left(\mathrm{CDCl}_{3}, 250 \mathrm{MHz}\right) \delta 6.49$ 6.85(1H, m), 7.25-7.29 (3H, m), 7.39 (1H, m), 7.68 (2H, d, J= $5.0 \mathrm{~Hz}), 7.77$ (1H, s), 8.18 (1H, s); ${ }^{13}$ C-NMR $\left(\mathrm{CDCl}_{3}, 63 \mathrm{MHz}\right) \delta 108.62(\mathrm{CH}), 115.46$ (quat), $125.55(\mathrm{CH}), 128.06$ (CH), $128.68(\mathrm{CH}), 130.89$ (quat), 132.58 (CH), 141.54 (quat), 142.64 (CH), 143.85 (CH), 157.09 (quat); HRMS (ES) calculated for $\mathrm{C}_{13} \mathrm{H}_{9} \mathrm{NO}_{2} 212.0706$; found 212.0704 .

General procedure for the synthesis of 4,4-dioxazoles: The following procedure for the preparation of compound 13 is representative.

- 2-Phenyl-4-(2-phenyloxazol-4-yl)oxazole 10

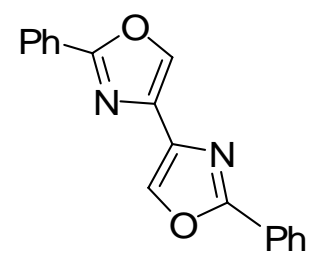

2-Phenyl-4-(2-phenyloxazol-4-yl)oxazole was prepared in a 2 step one pot procedure: A microwave vial was charged with 2-phenyloxazol-4-yl trifluoromethanesulfonate (119 $\mathrm{mg}, 0.41 \mathrm{mmol}, 1.2$ equiv), bis(pinacolato)diboron (116 mg, $0.45 \mathrm{mmol}, 1.3$ equiv), $\mathrm{PdCl}_{2}$ (dppf) (3 mol \%), dppf (3 mol \%), sodium acetate (102 mg, $1.22 \mathrm{mmol}, 3$ equiv) and 1,4 dioxane (5 mL). The mixture was then irradiated in a Smith synthesiser for 20 minutes at a pre-selected temperature of $150{ }^{\circ} \mathrm{C}$. After the reaction the vial was cooled with air jet cooling to room temperature. The vial was opened and 2-phenyloxazol-4-yl trifluoromethanesulfonate (100 mg, 0.34 mmol, 1 equiv), $\mathrm{PdCl}_{2}\left(\mathrm{PPh}_{3}\right)_{4}(5 \mathrm{~mol} \%)$ and sodium carbonate $2 \mathrm{M}(0.53 \mathrm{~mL}, 1.06 \mathrm{mmol}, 3$ equiv) were added to 
the reaction mixture. The vial was resealed and the mixture was again irradiated for 20 minutes at a preselected temperature of $150{ }^{\circ} \mathrm{C}$. The vial was cooled with air jet cooling and the crude mixture was filtered through a pad of celite ${ }^{\circledR}$ with thorough acetone washing. The organic layers were concentrated in vacuo and the residue was purified by flash chromatography (silica, hexanes/DCM 5:5) to give the bis-oxazole product 13 (57 mg, $58 \%$ yield) as a white solid; $\mathrm{mp}=110-11{ }^{\circ} \mathrm{C} ;{ }^{1} \mathbf{H}-\mathbf{N M R}\left(\mathrm{CDCl}_{3}, 360 \mathrm{MHz}\right): \delta$ 7.46-7.50 (6H, m), 8.08-8.13 (6H, m); ${ }^{13}$ C-NMR $\left(\mathrm{CDCl}_{3}, 90 \mathrm{MHz}\right) \delta 126.58(\mathrm{CH}), 127.19$ (quat), 128.78 (CH), $130.60(\mathrm{CH}), 134.75$ (quat), 134.99 (CH), 162.16 (quat). HRMS (ES) calculated for $\mathrm{C}_{18} \mathrm{H}_{12} \mathrm{~N}_{2} \mathrm{O}_{2}$ 289.0972; found 289.0971.

- 2-(4-Fluorophenyl)-4-(2-(4-fluorophenyl)oxazol-4-yl)oxazole 11

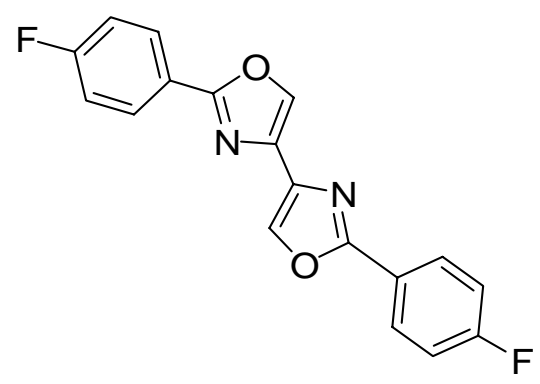

Prepared according to the general procedure. Purification by flash chromatography (silica, hexanes/EtOAc 8:2) gave a white solid which was further purified by trituration from hexane to afford the dioxazole product (72 mg, $69 \%$ yield) as a white solid; mp = 258-259 ${ }^{\circ} \mathrm{C}$; ${ }^{1} \mathbf{H}-\mathrm{NMR}$ (DMSO, $360 \mathrm{MHz}$ ): 7.40-7.45 (4H, m), 8.07-8.11 (4H, m), 8.59 (2H, s); ${ }^{13} \mathrm{C}-\mathrm{NMR}$ (DMSO, $\left.90 \mathrm{MHz}\right) \delta 115.55$ (CH, d, $J=22.0 \mathrm{~Hz}$ ), 122.82 (q, d, J= 2.7 Hz), 128.18 (CH, d, $J=9.0 \mathrm{~Hz}$ ), 133.60 (quat), 135.59 (CH), 160.12 (quat), 163.15 (quat, d, $J=247.0 \mathrm{~Hz}$ ); HRMS (ES) calculated for $\mathrm{C}_{18} \mathrm{H}_{10} \mathrm{~N}_{2} \mathrm{O}_{2} \mathrm{~F}_{2}$ 325.0783; found 325.0783.

- 2-(4-Methoxyphenyl)-4-(2-(4-methoxyphenyl)oxazol-4-yl)oxazole 12

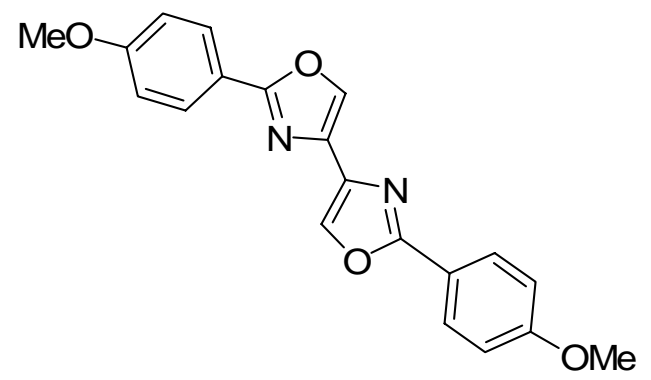

Prepared according to the general procedure. Purification by flash chromatography (silica, hexanes/EtOAc 7:3) gave the dioxazole product (78 mg, $61 \%$ yield) as a white solid; $\mathrm{mp}=180{ }^{\circ} \mathrm{C}$ (decomp.); ${ }^{1} \mathbf{H}-\mathbf{N M R}\left(\mathrm{CDCl}_{3}, 250\right.$ 
MHz): $3.88\left(\mathrm{CH}_{3}, \mathrm{~s}\right), 6.99(4 \mathrm{H}, \mathrm{d}, J=10.0 \mathrm{~Hz}), 8.04(4 \mathrm{H}, \mathrm{d}, J=10.0 \mathrm{~Hz}), 8.07(2 \mathrm{H}, \mathrm{s}) .{ }^{13} \mathrm{C}-\mathbf{N M R}\left(\mathrm{CDCl}_{3}, 63\right.$ MHz) $\delta 55.39\left(\mathrm{CH}_{3}\right), 114.17(\mathrm{CH}), 120.01$ (quat), $128.26(\mathrm{CH}), 134.37(\mathrm{CH}), 134.57$ (quat), 161.51 (quat), 162.20 (quat). HRMS (ES) calculated for $\mathrm{C}_{20} \mathrm{H}_{16} \mathrm{~N}_{2} \mathrm{O}_{4} 349.1183$; found 349.1187.

- 2-(4-Fluorophenyl)-4-(2-phenyloxazol-4-yl)oxazole 13

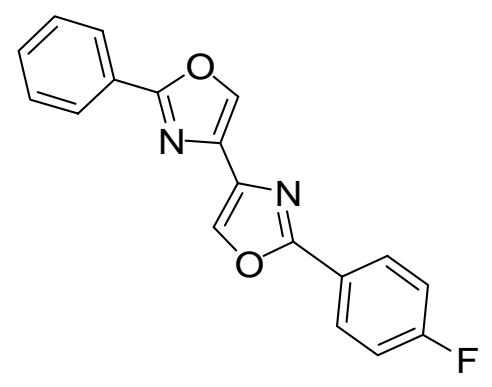

Prepared according to the general procedure. Purification by flash chromatography (silica, hexanes/EtOAc 9:1) gave the dioxazole product (44 mg, 38\% yield) as a white solid; $\mathrm{mp}=184-186{ }^{\circ} \mathrm{C} ;{ }^{1} \mathbf{H}-\mathbf{N M R}\left(\mathrm{CDCl}_{3}, 250 \mathrm{MHz}\right)$ : 7.13-7.20 (2H, m), 7.46-7.49 (3H, m), 8.06-8.13 (6H, m); ${ }^{13}$ C-NMR (CDCl, $\left.90 \mathrm{MHz}\right) \delta 115.92(\mathrm{CH}, \mathrm{d}, J=22$ $\mathrm{Hz}), 123.48$ (quat), 126.54 (CH), 127.09 (quat), 128.66 (CH), 128.72 (CH), 128.75 (CH), 128.94 (quat), 130.57 (CH, d, $J=2.7 \mathrm{~Hz}$ ), 134.60 (q, d, $J=10.0 \mathrm{~Hz}$ ), 135.04 (CH), 161.35 (quat), 162.24 (quat), 164.2 (quat, d, $J=250$ $\mathrm{Hz}$ ); HRMS (FAB) calculated for $\mathrm{C}_{18} \mathrm{H}_{11} \mathrm{~N}_{2} \mathrm{O}_{2} \mathrm{~F}$ 307.08828; found 307.08821. 
Part B: Spectroscopic Data 


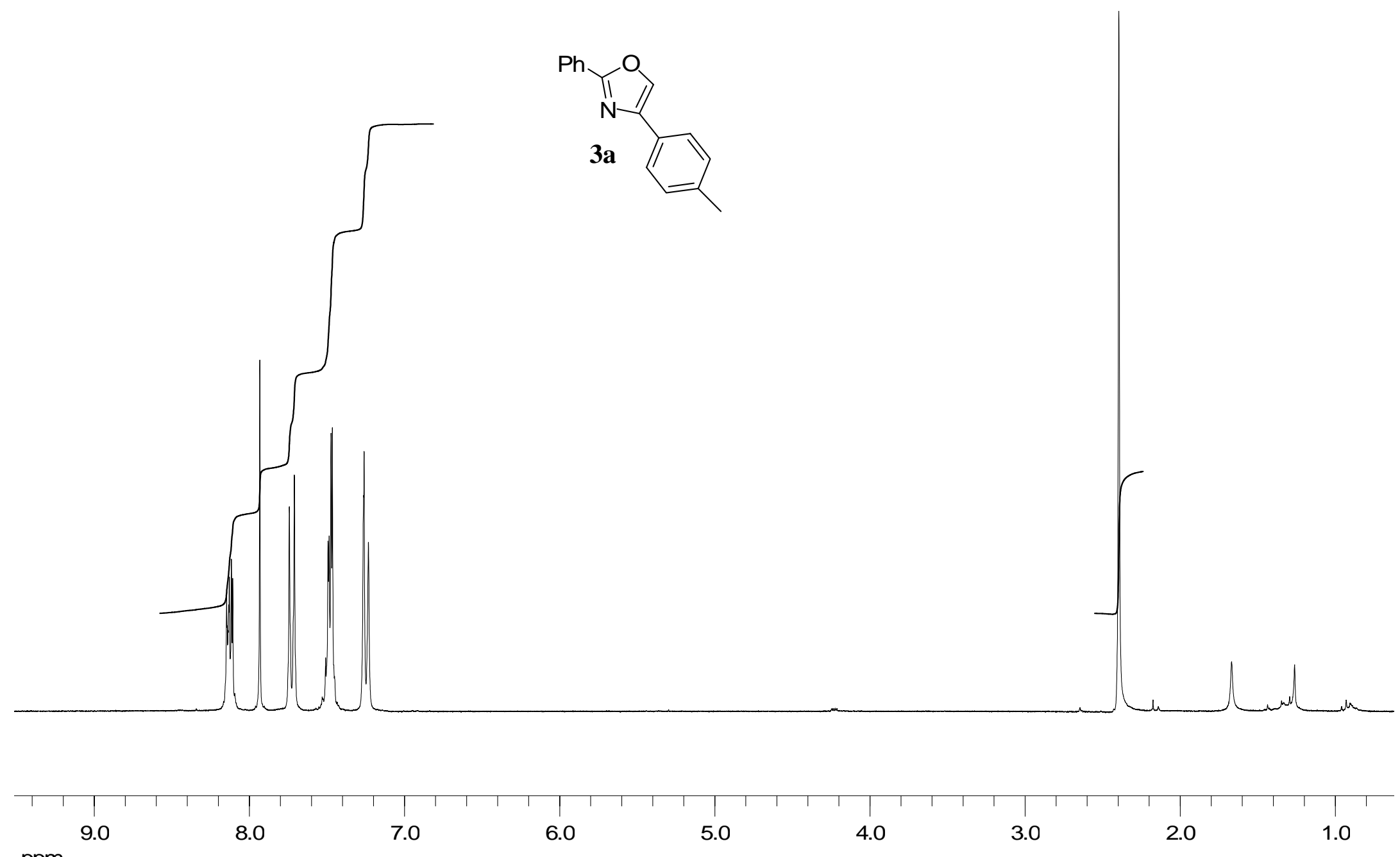

Page 16 

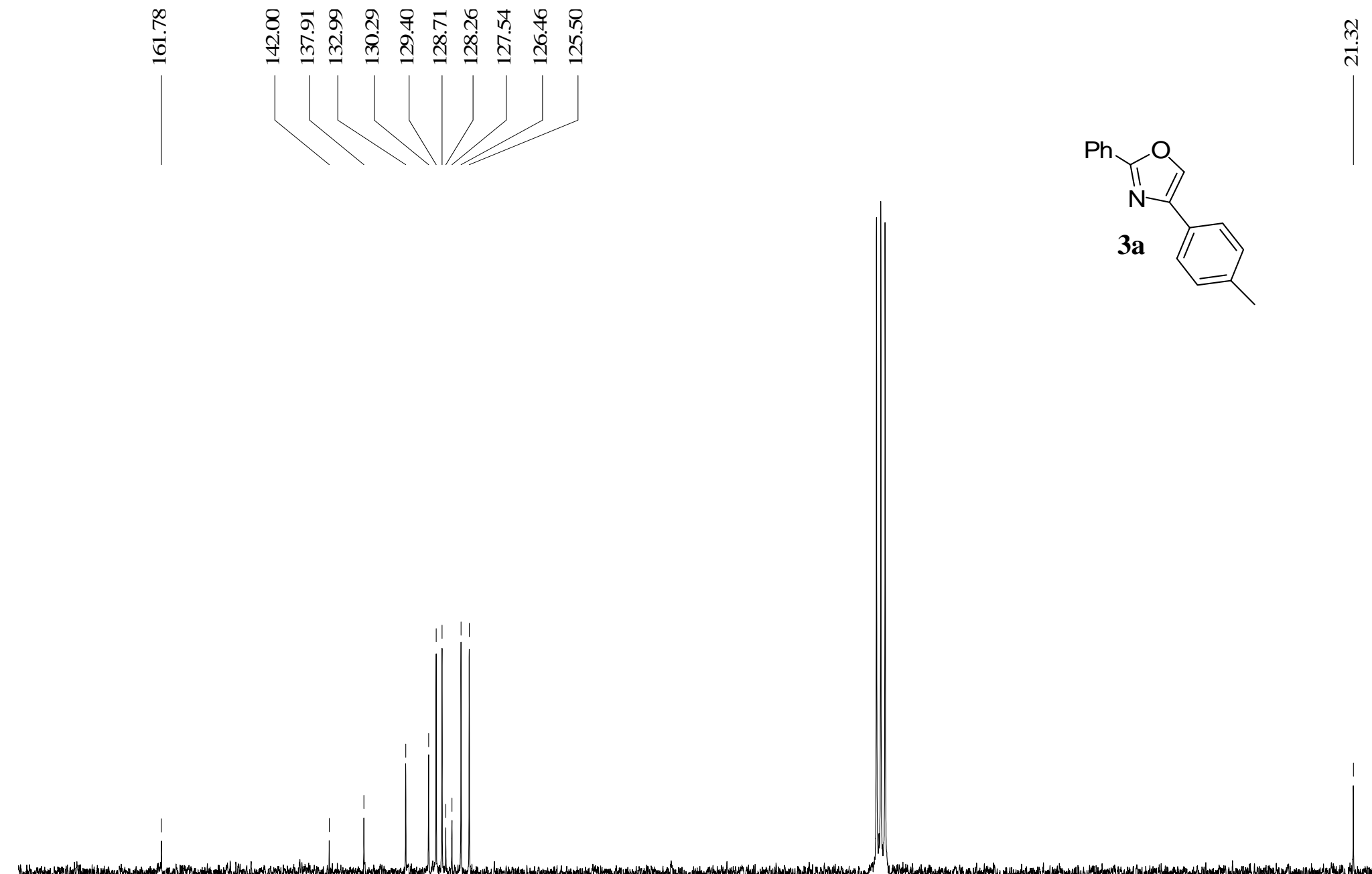

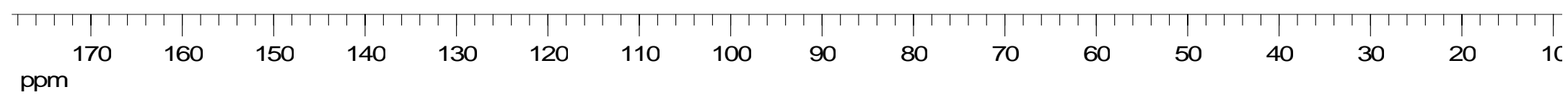




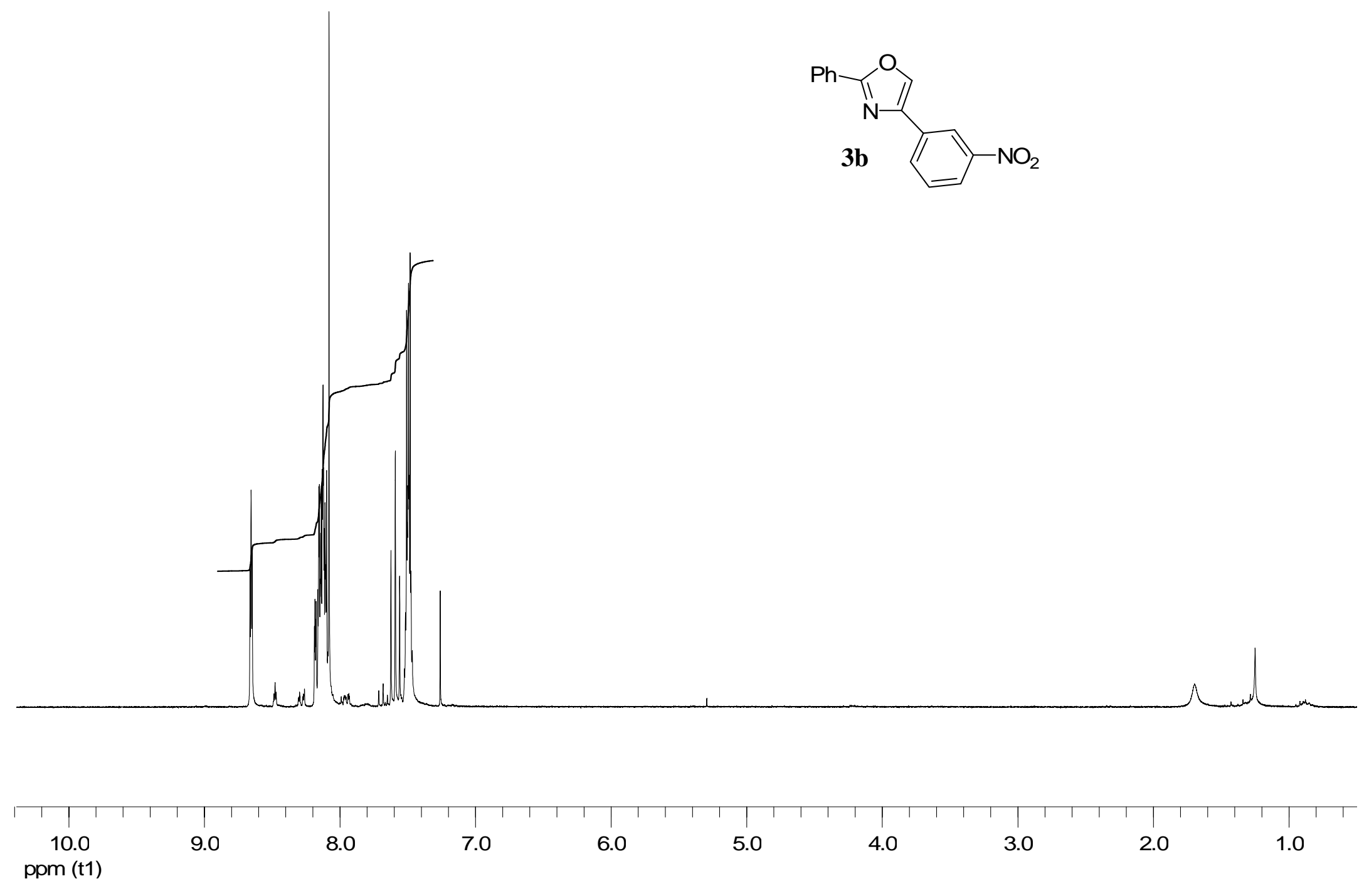

Page 18 

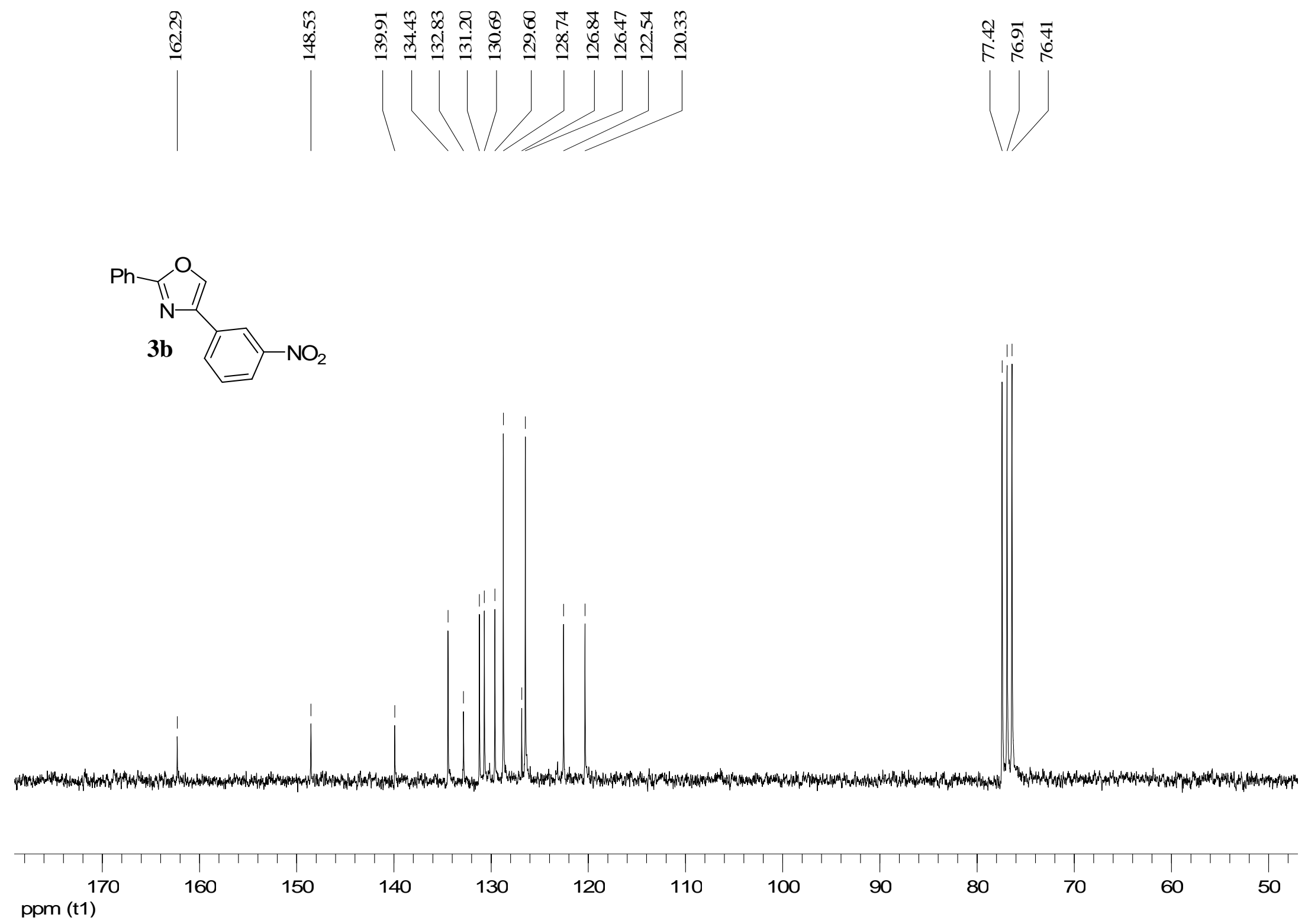


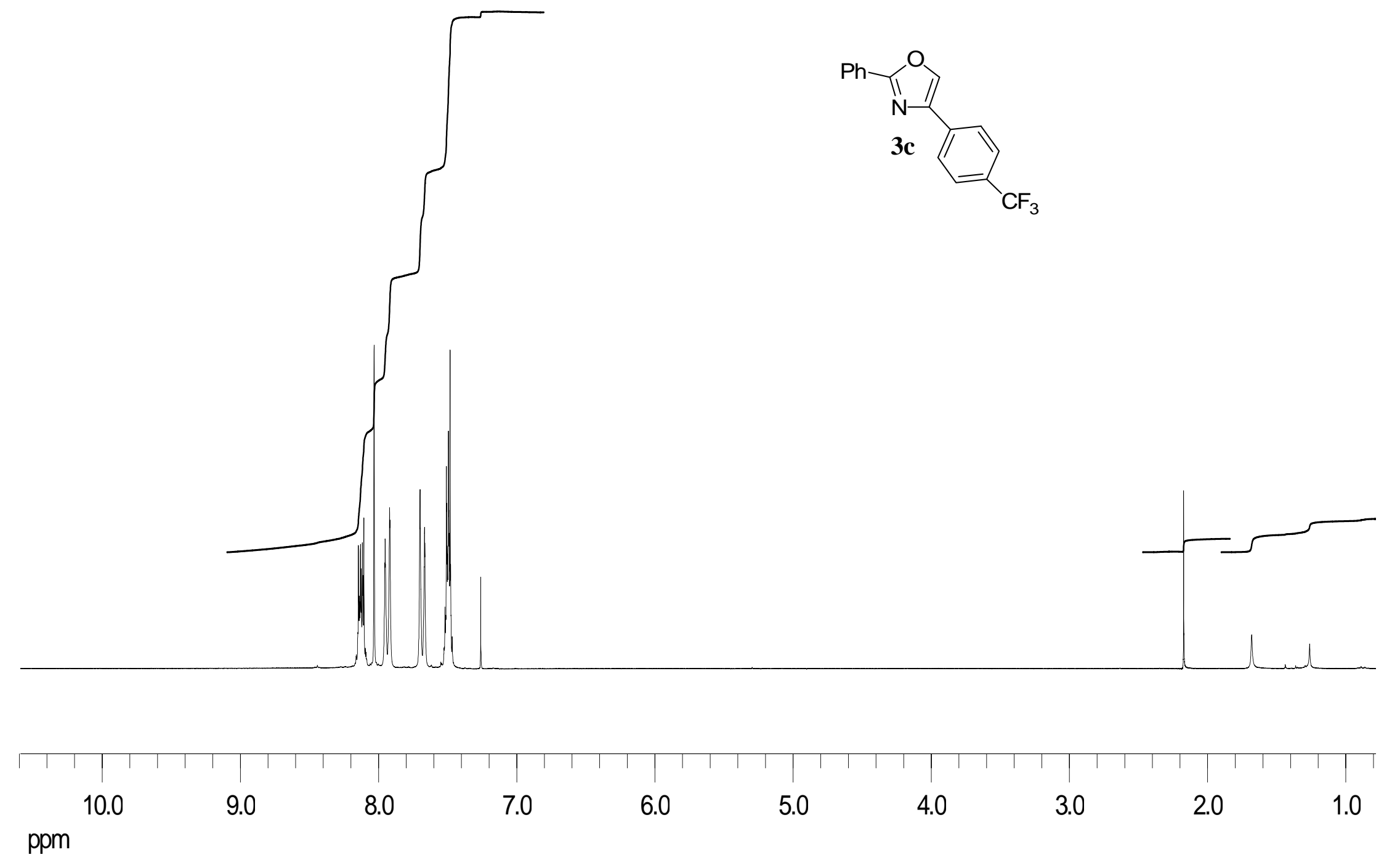



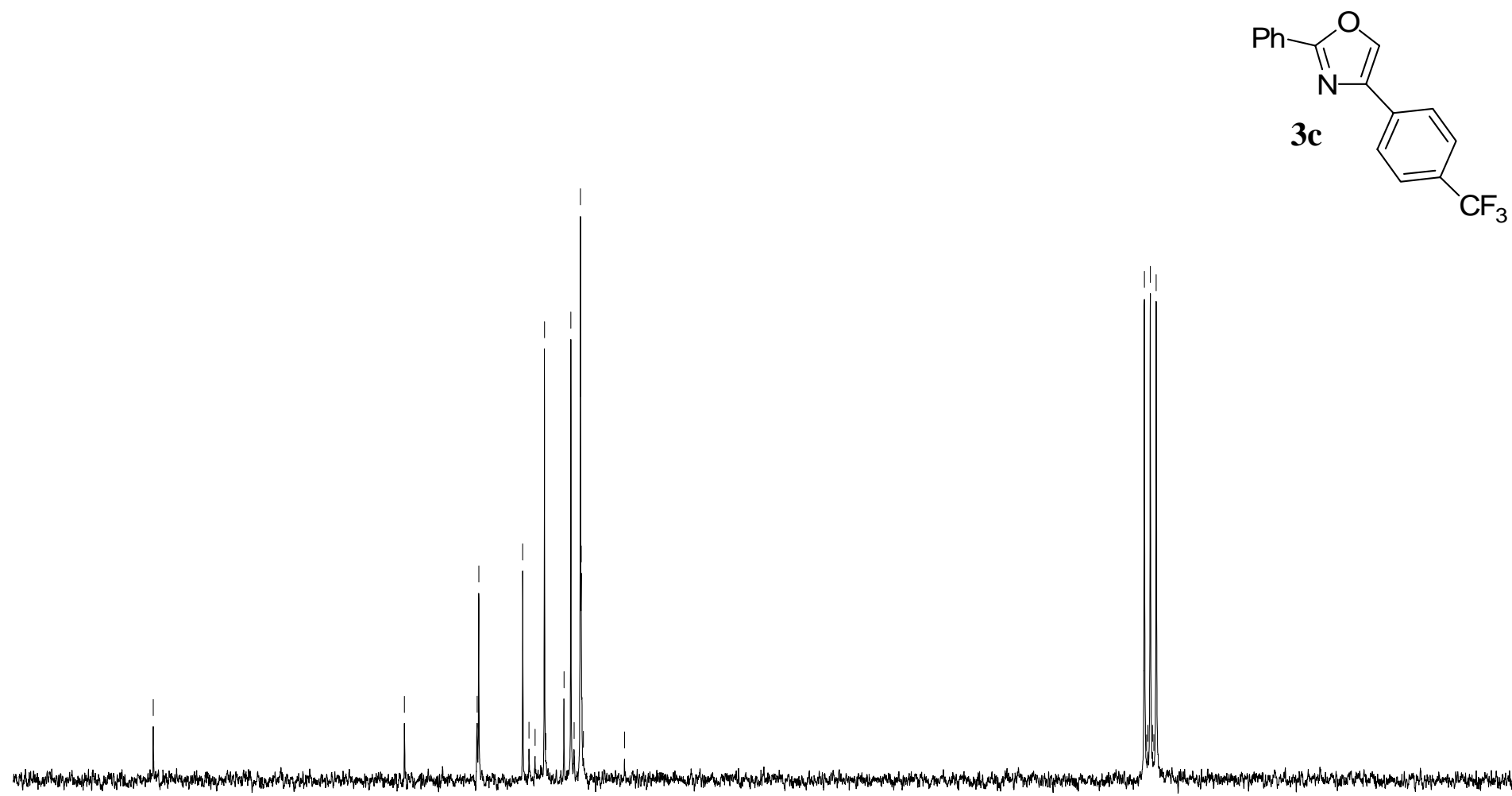

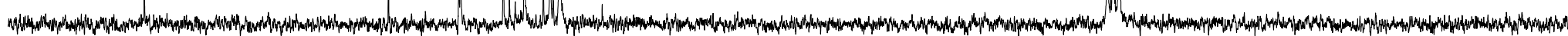

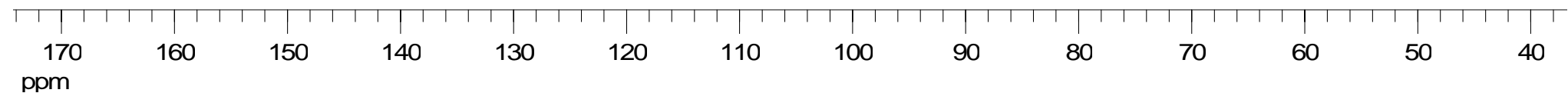




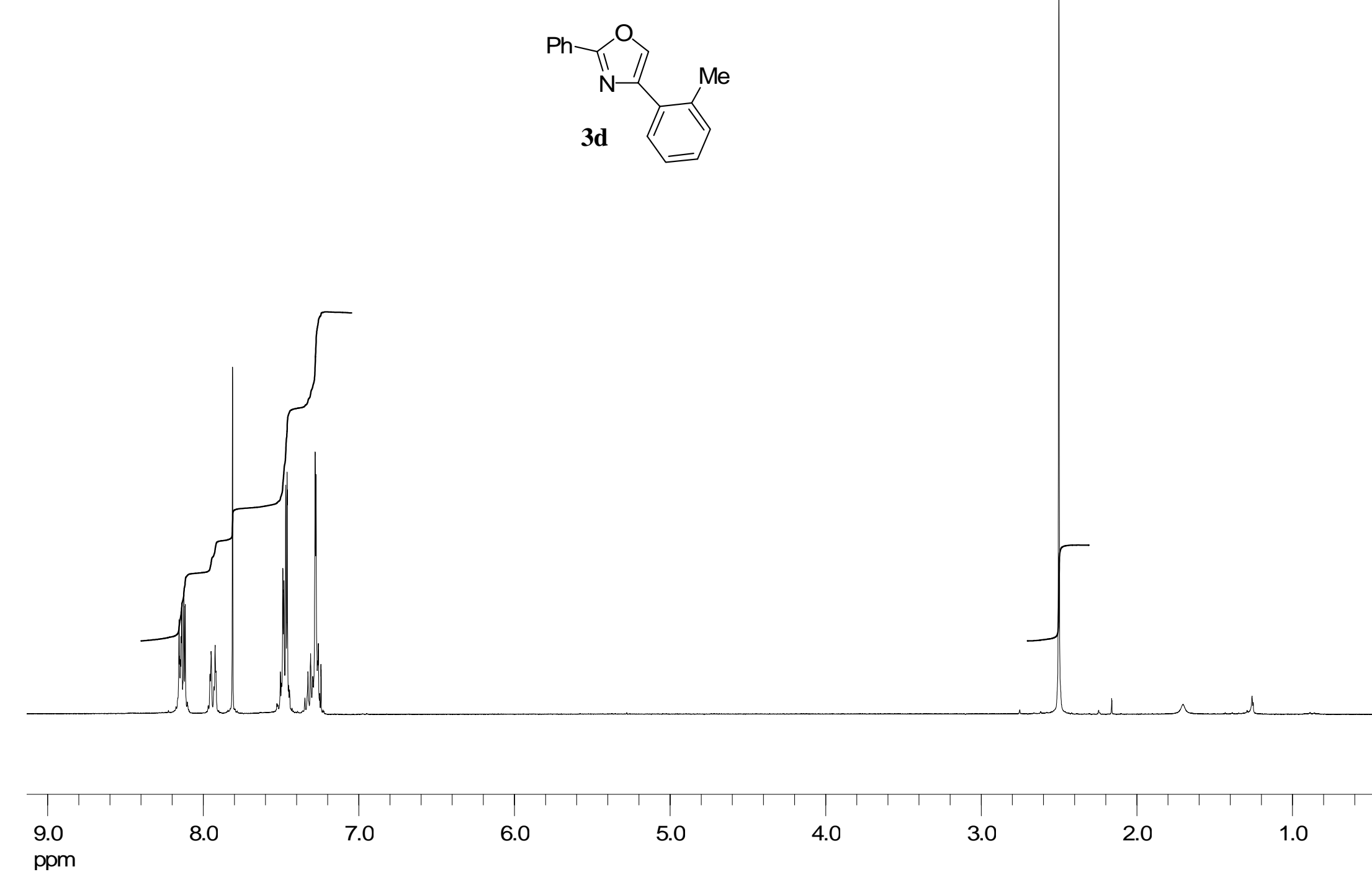

Page 22 


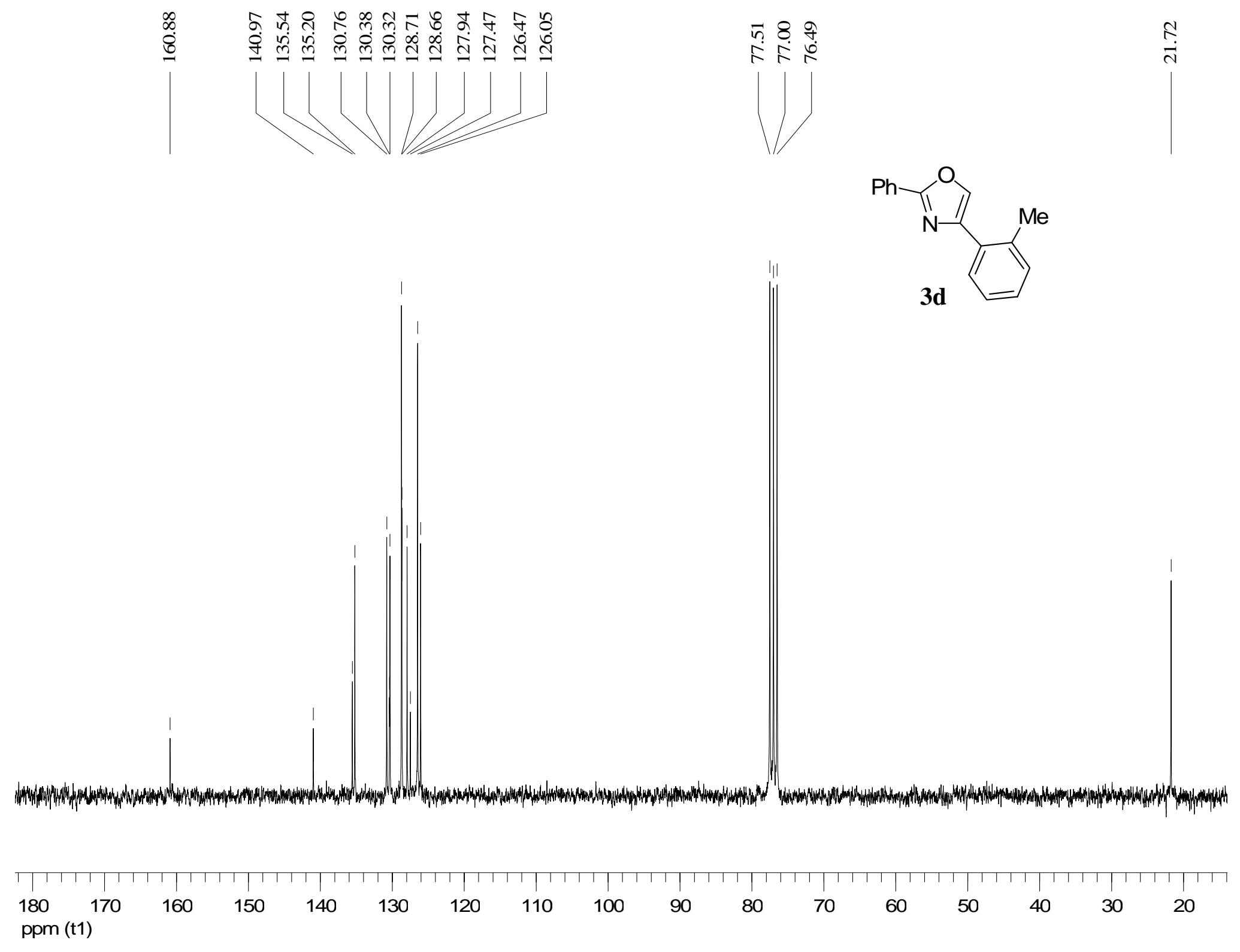



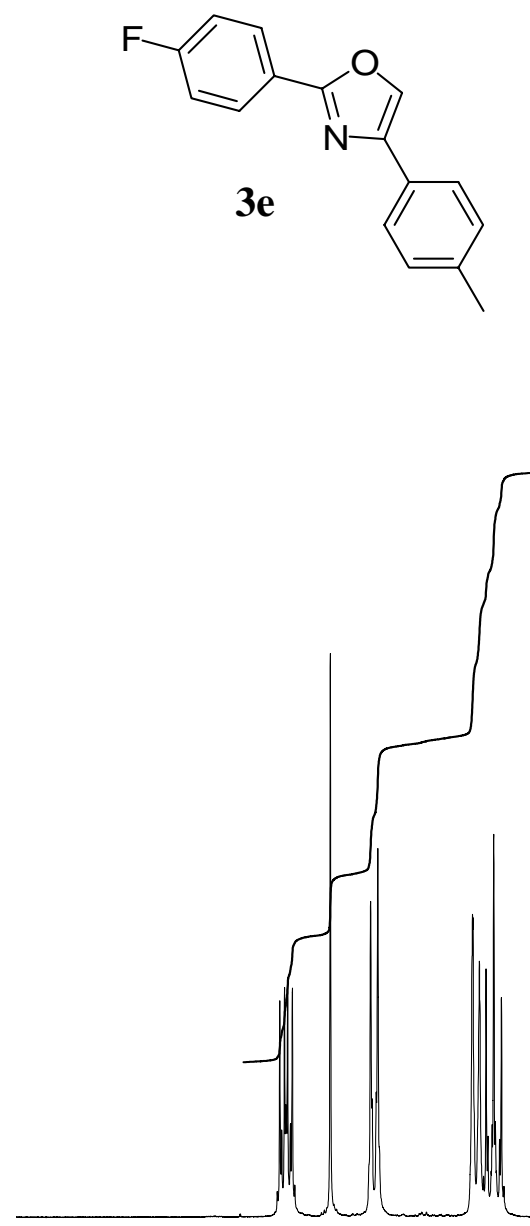

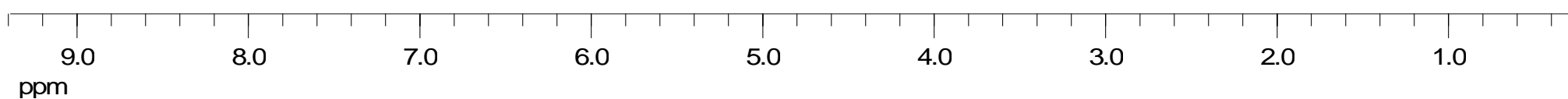

Page 24 

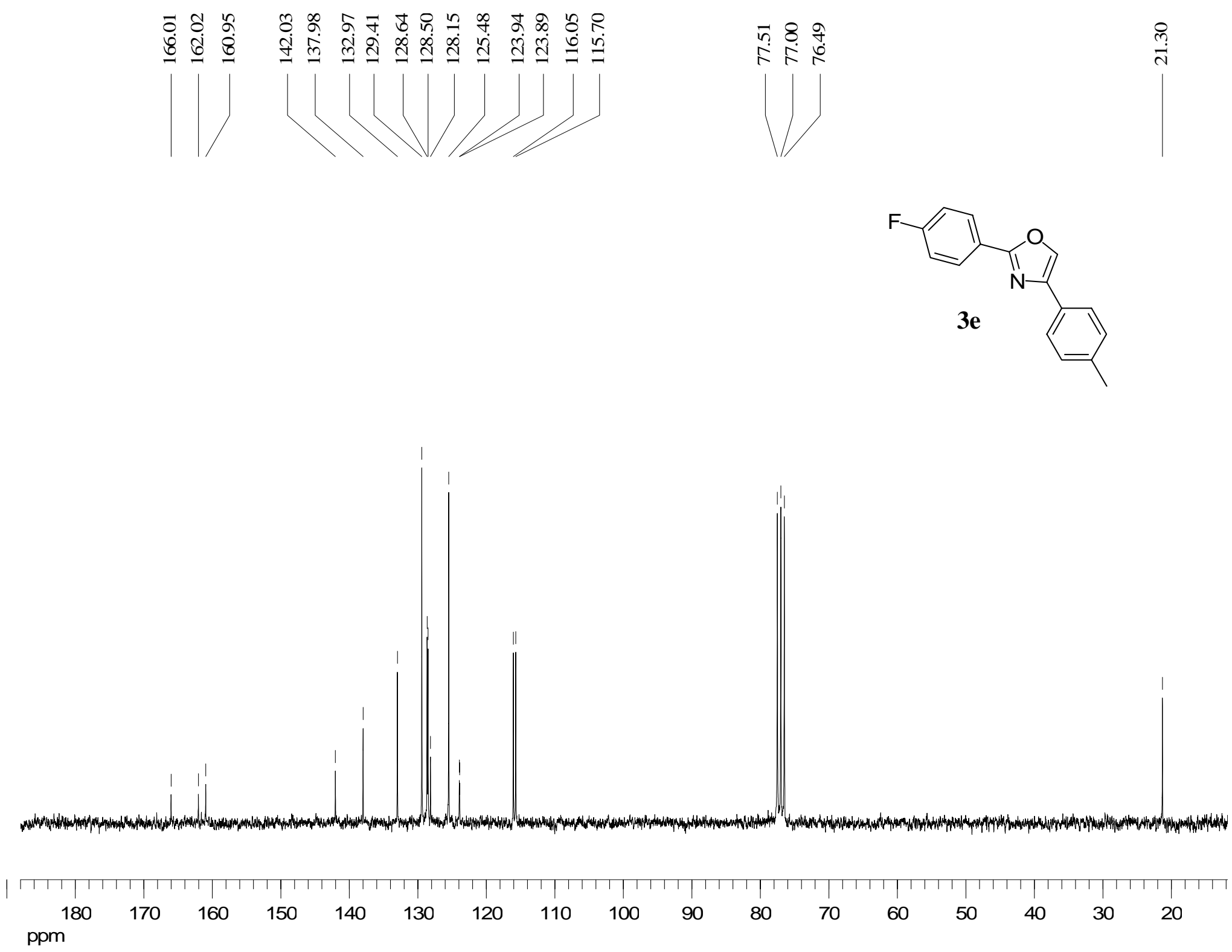


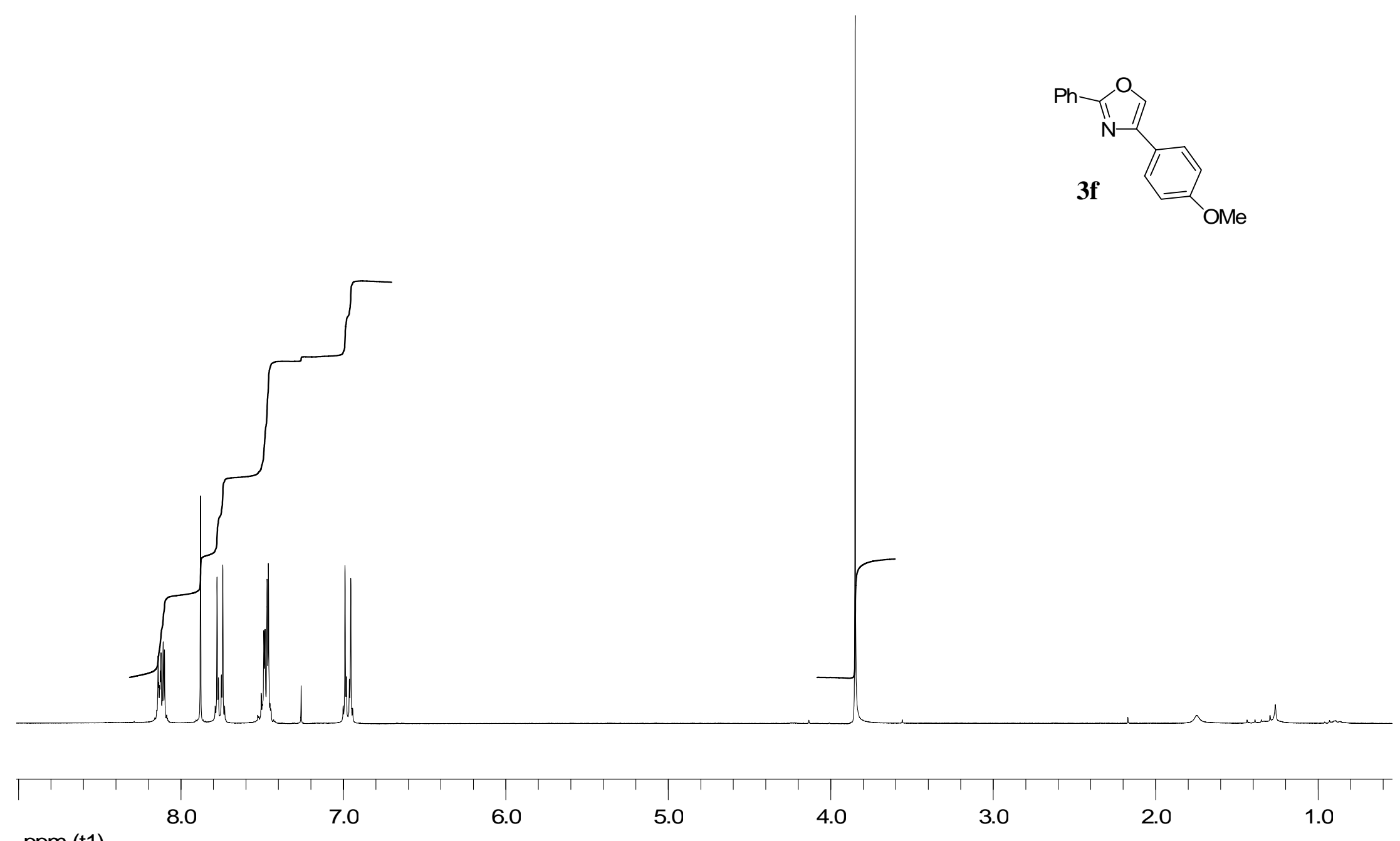

ppm (t1)

Page 26 

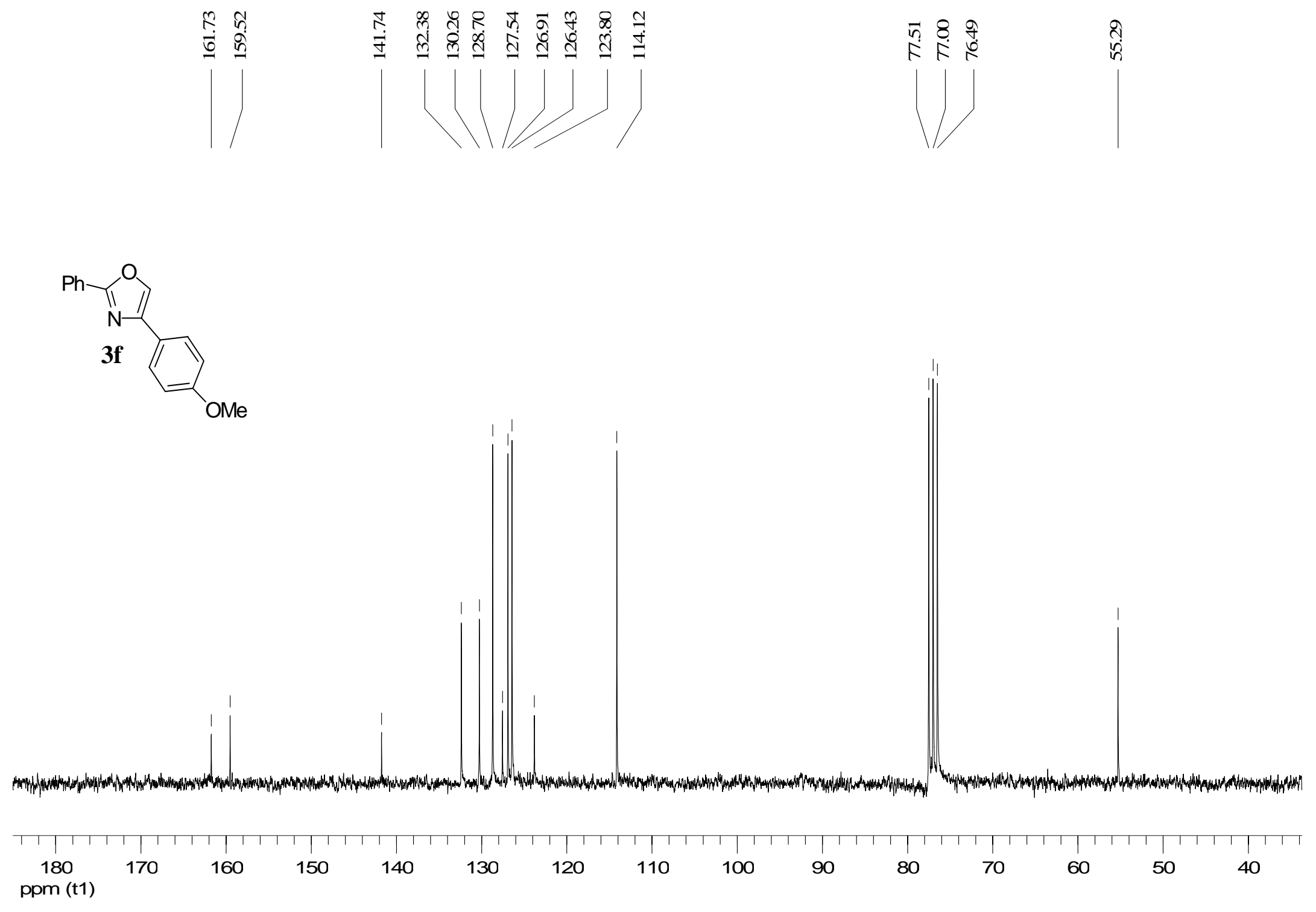

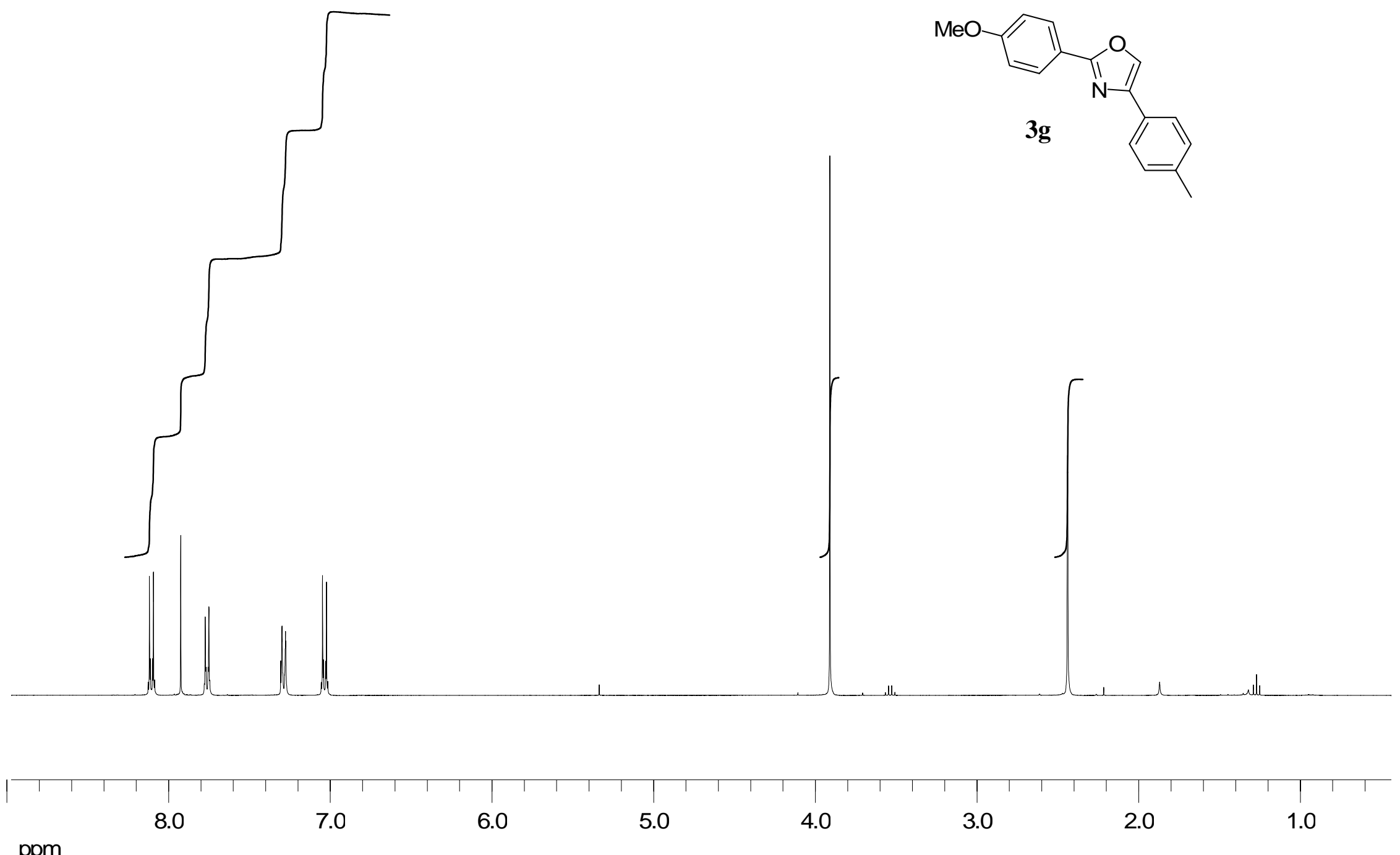

ppm

Page 28 

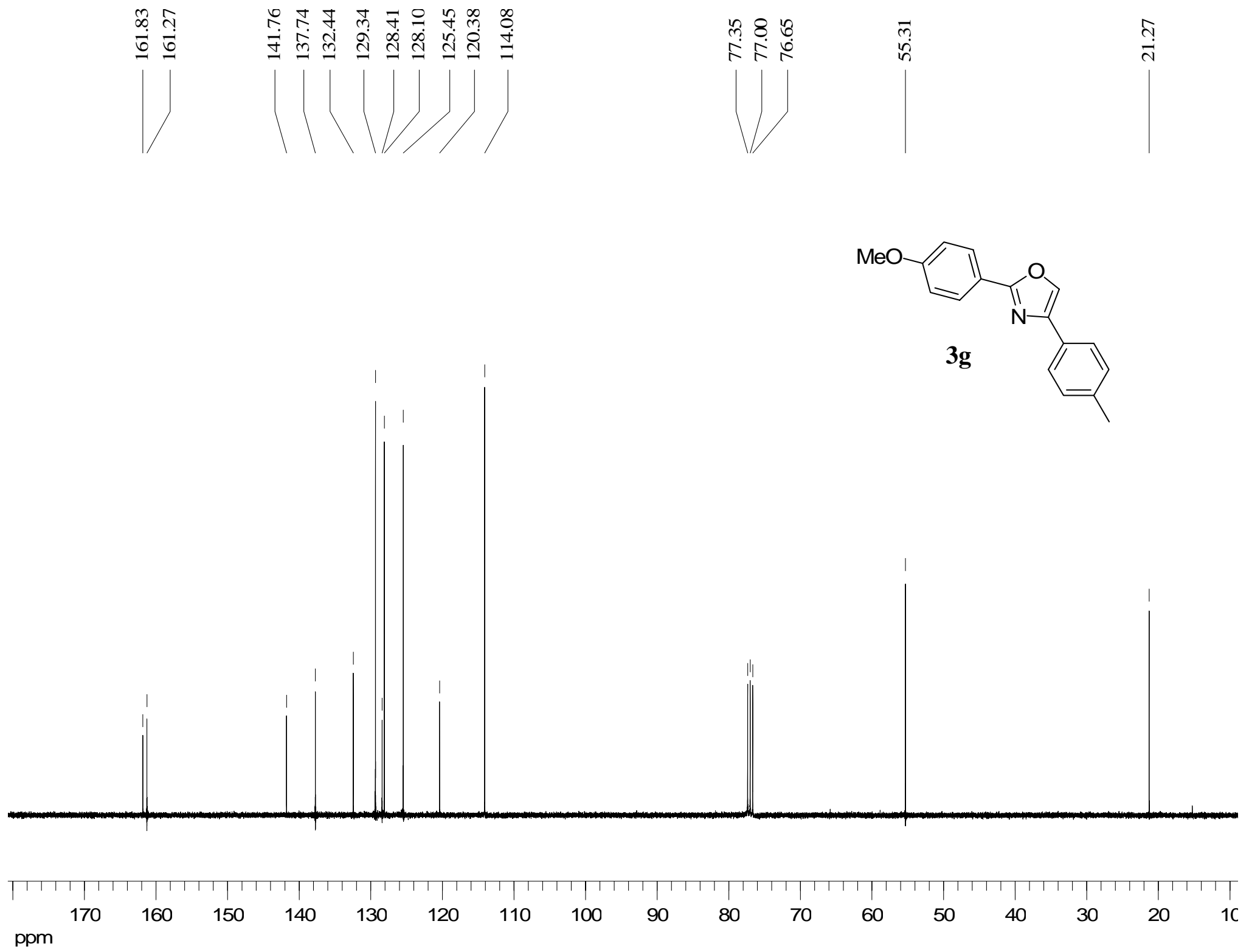


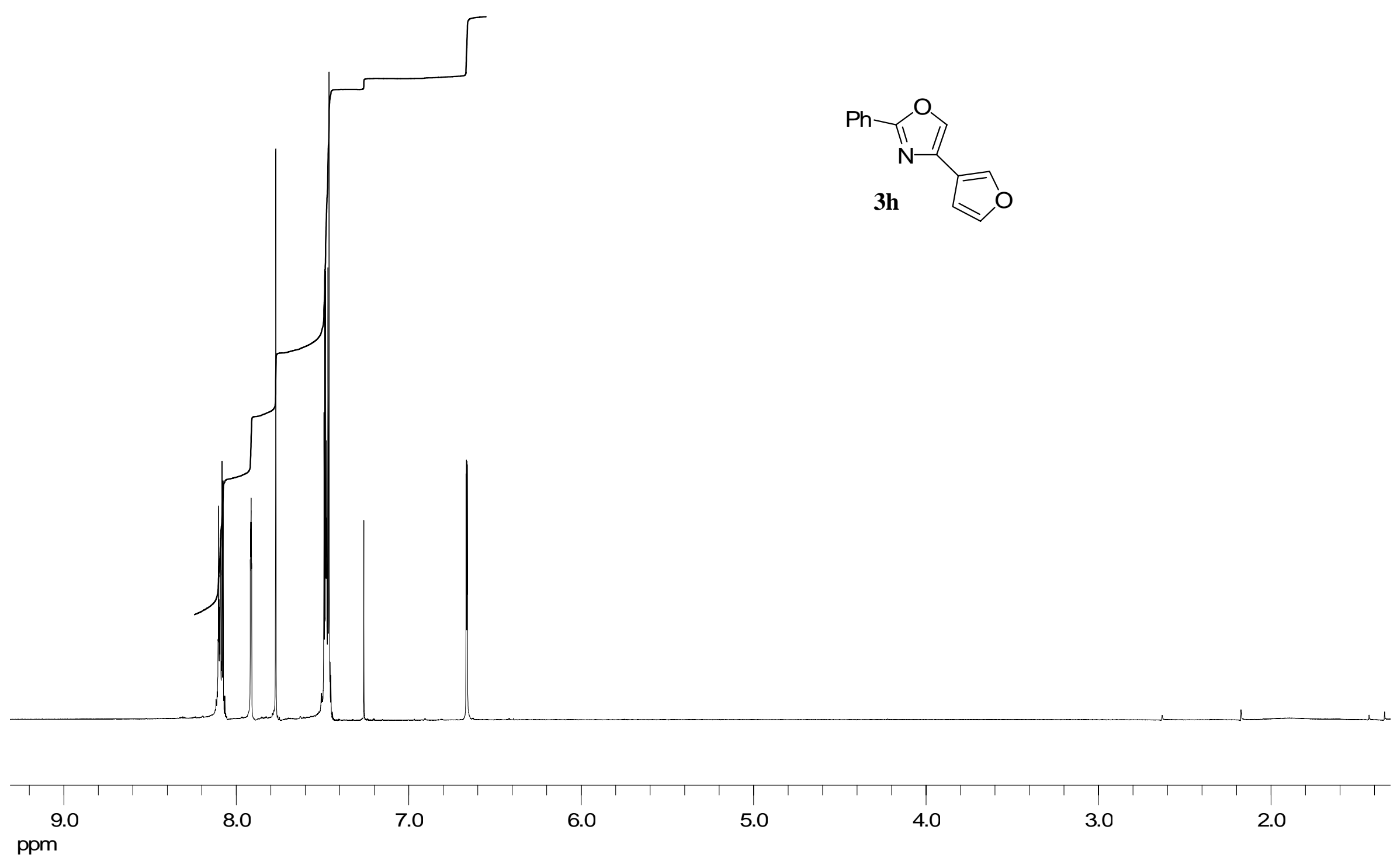

Page 30 

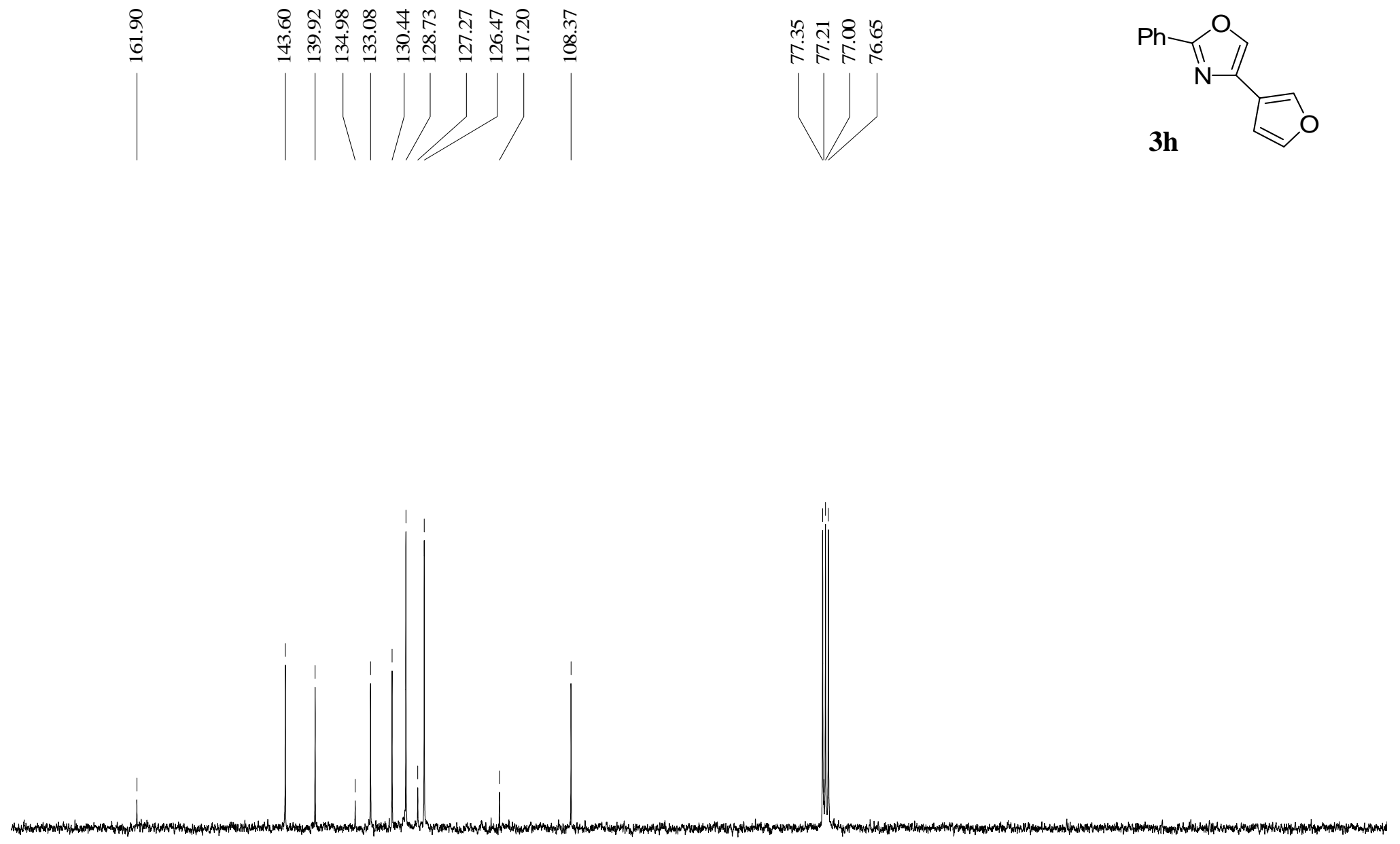

170
ppm



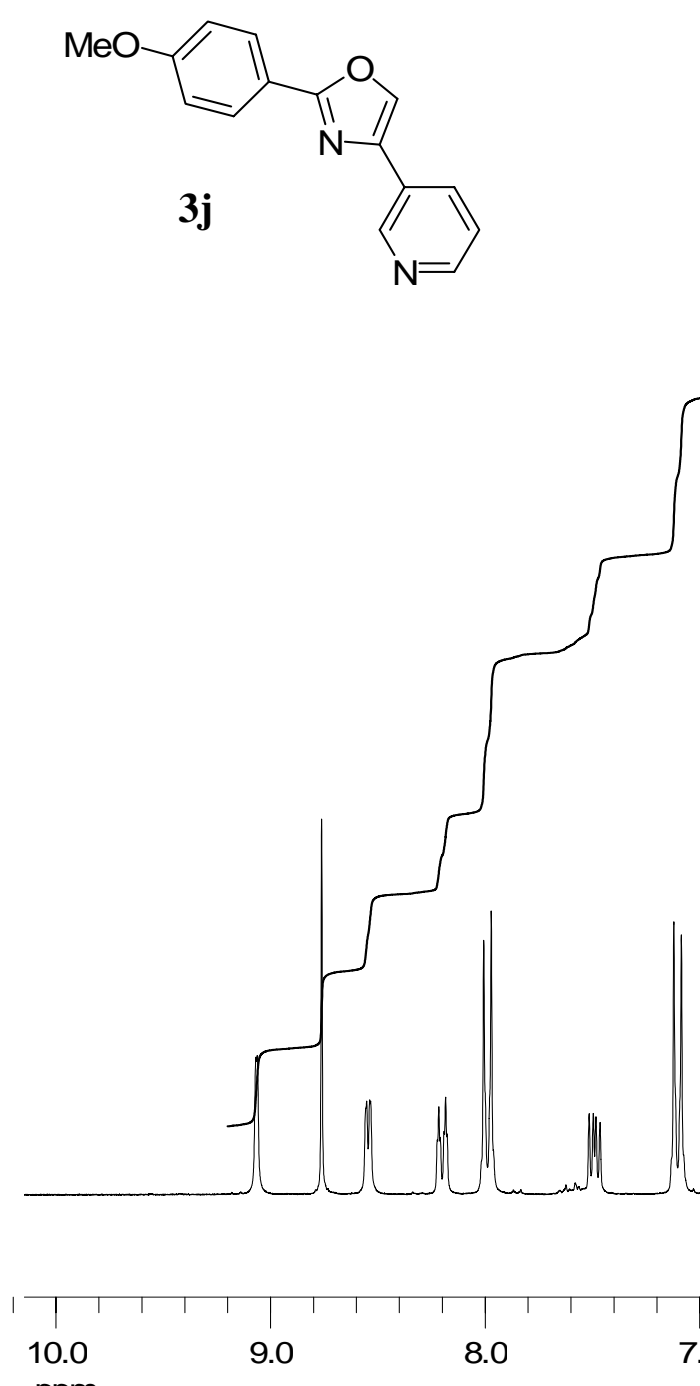

ppm

9.0

8.0

7.0

6.0

5.0

4.0

3.0

2.0

1.0 

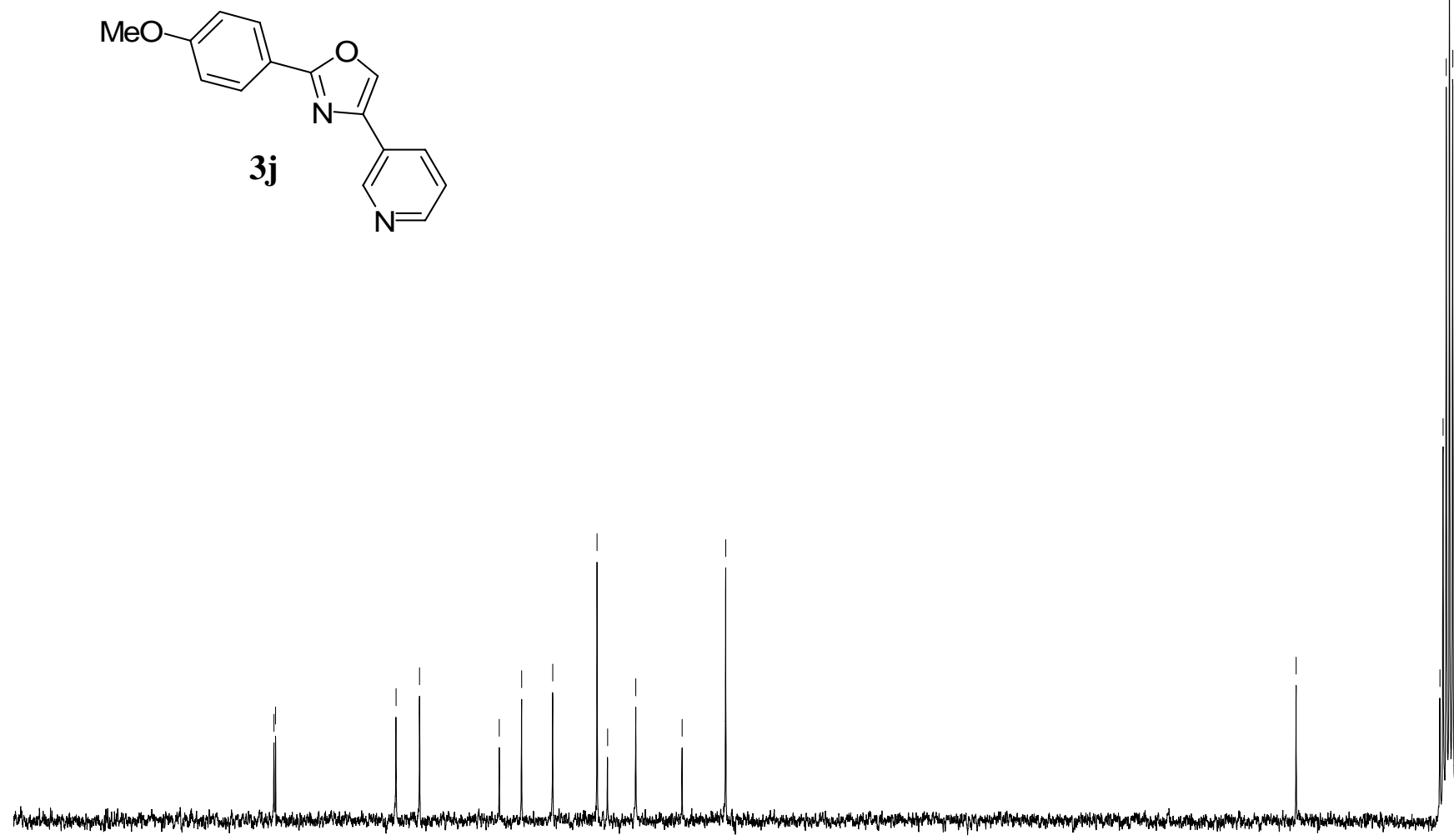

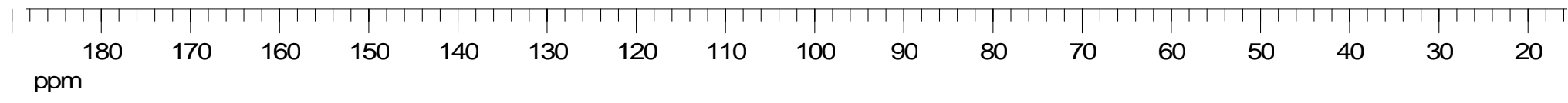




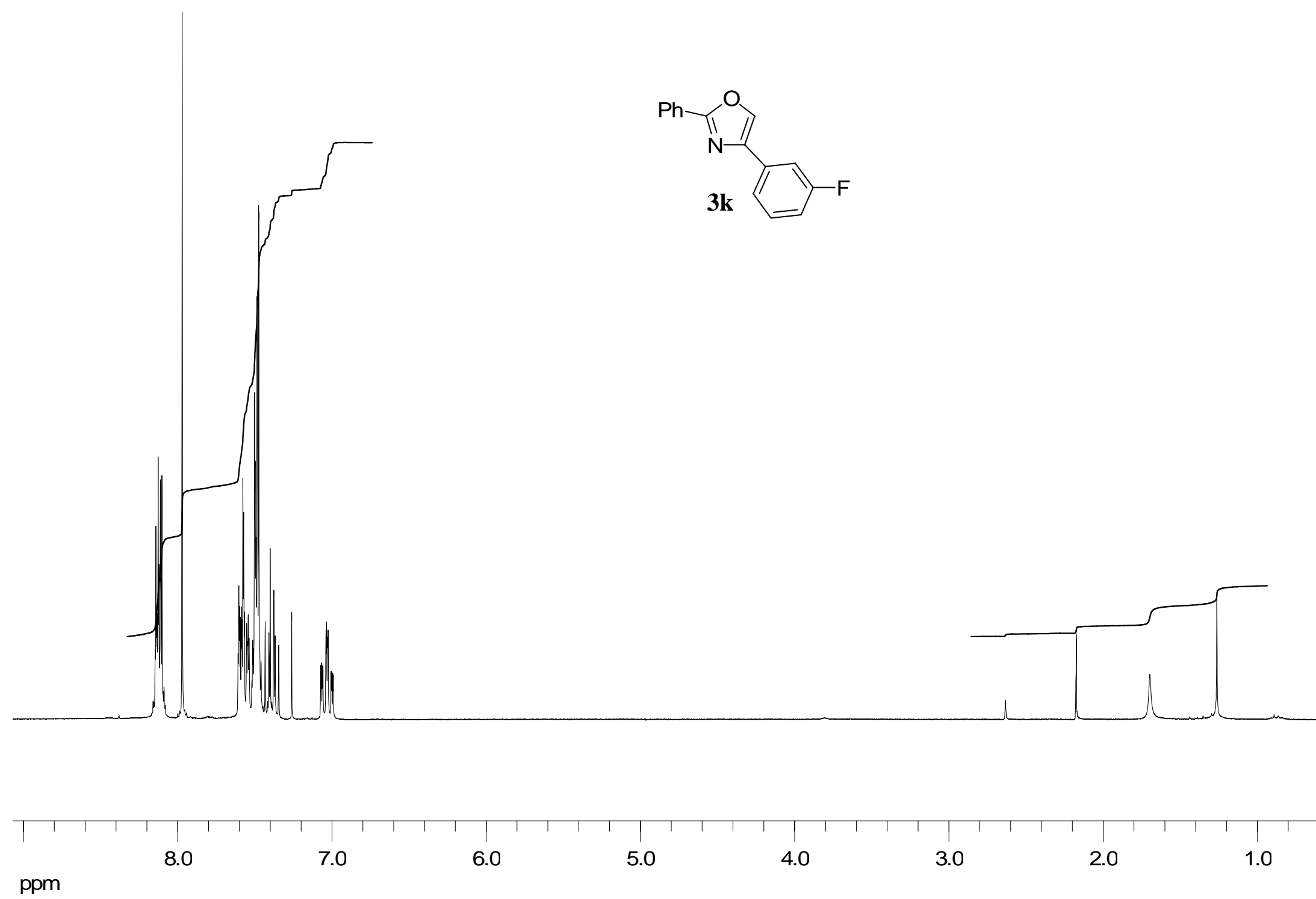

Page 34 

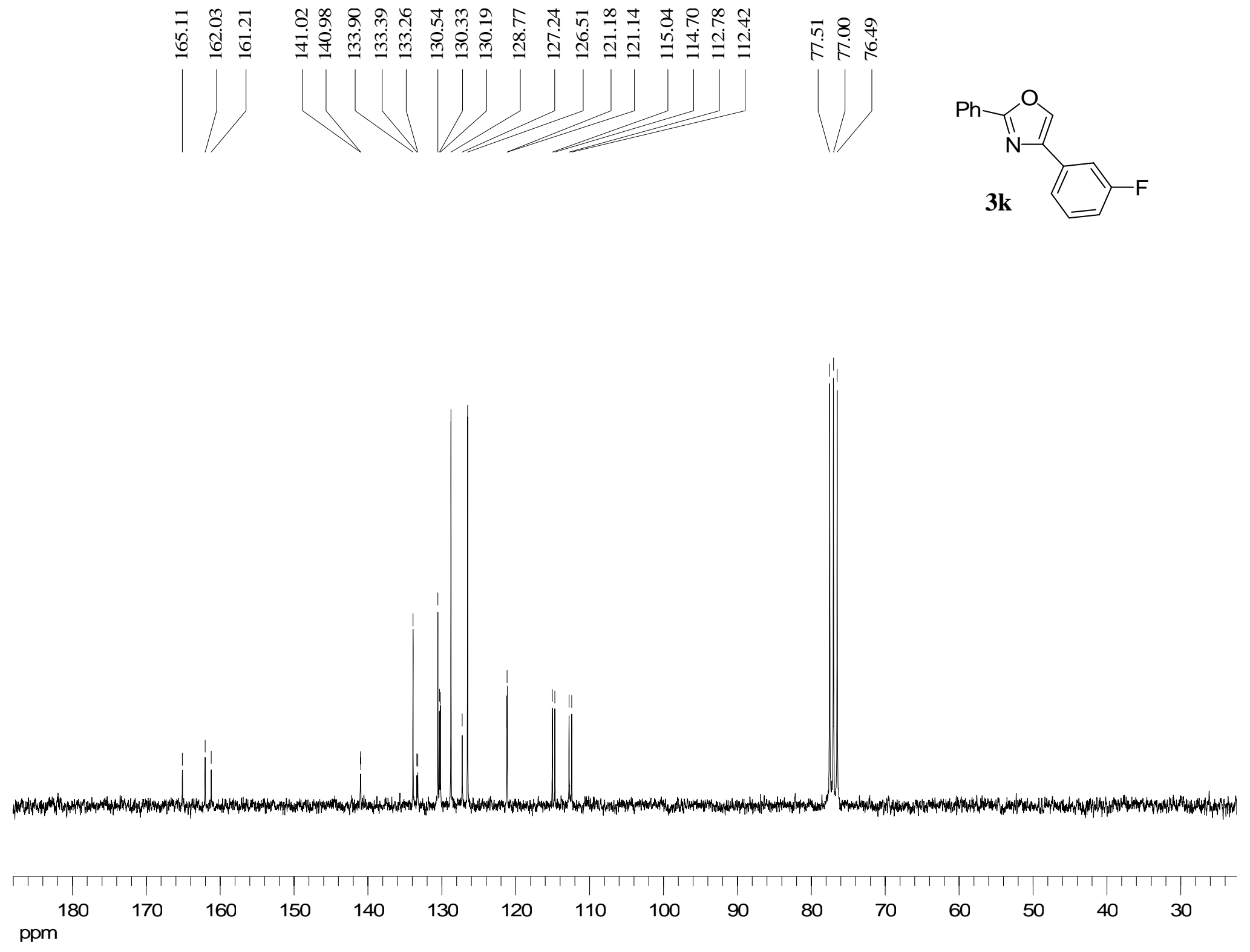


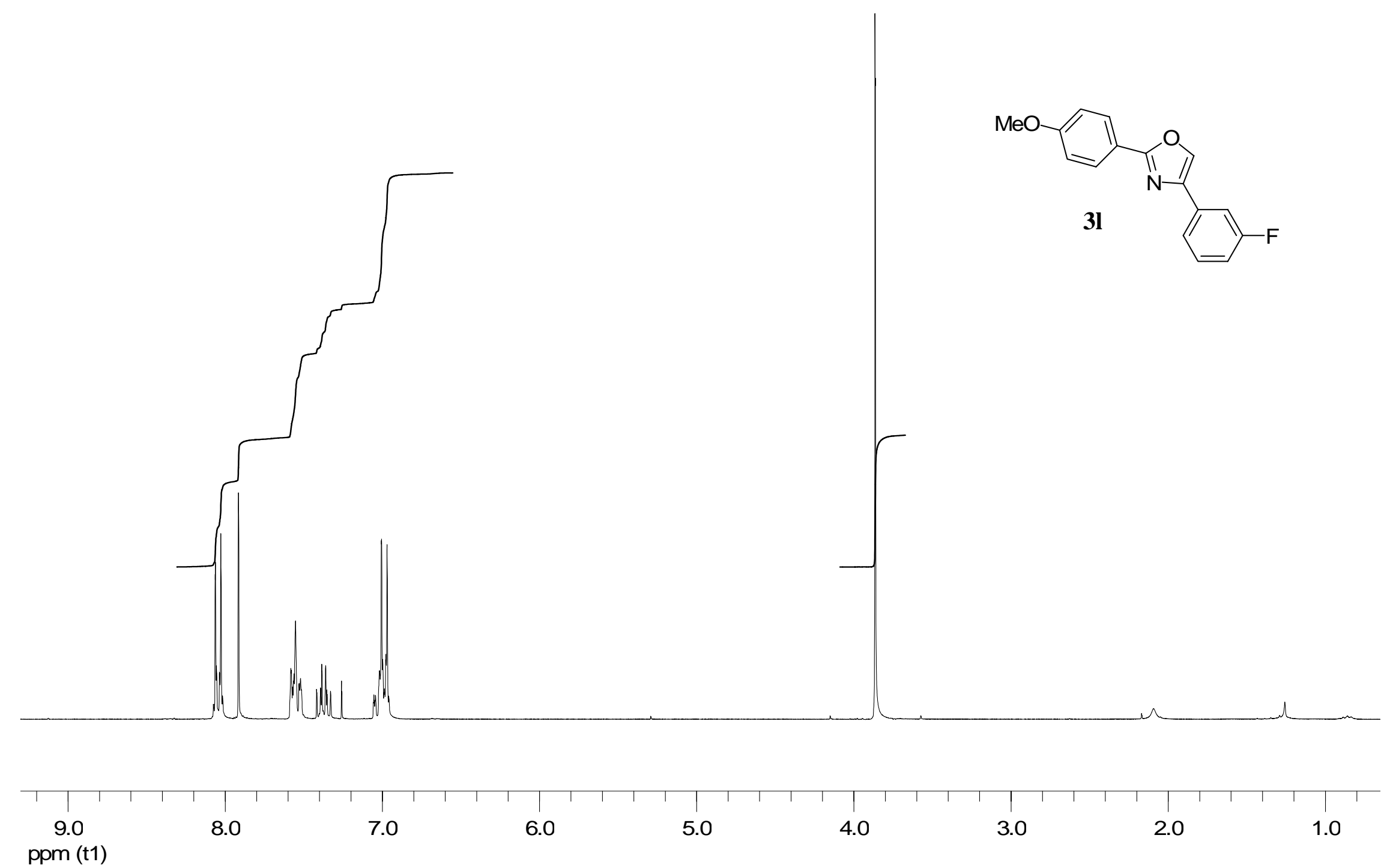

Page 36 

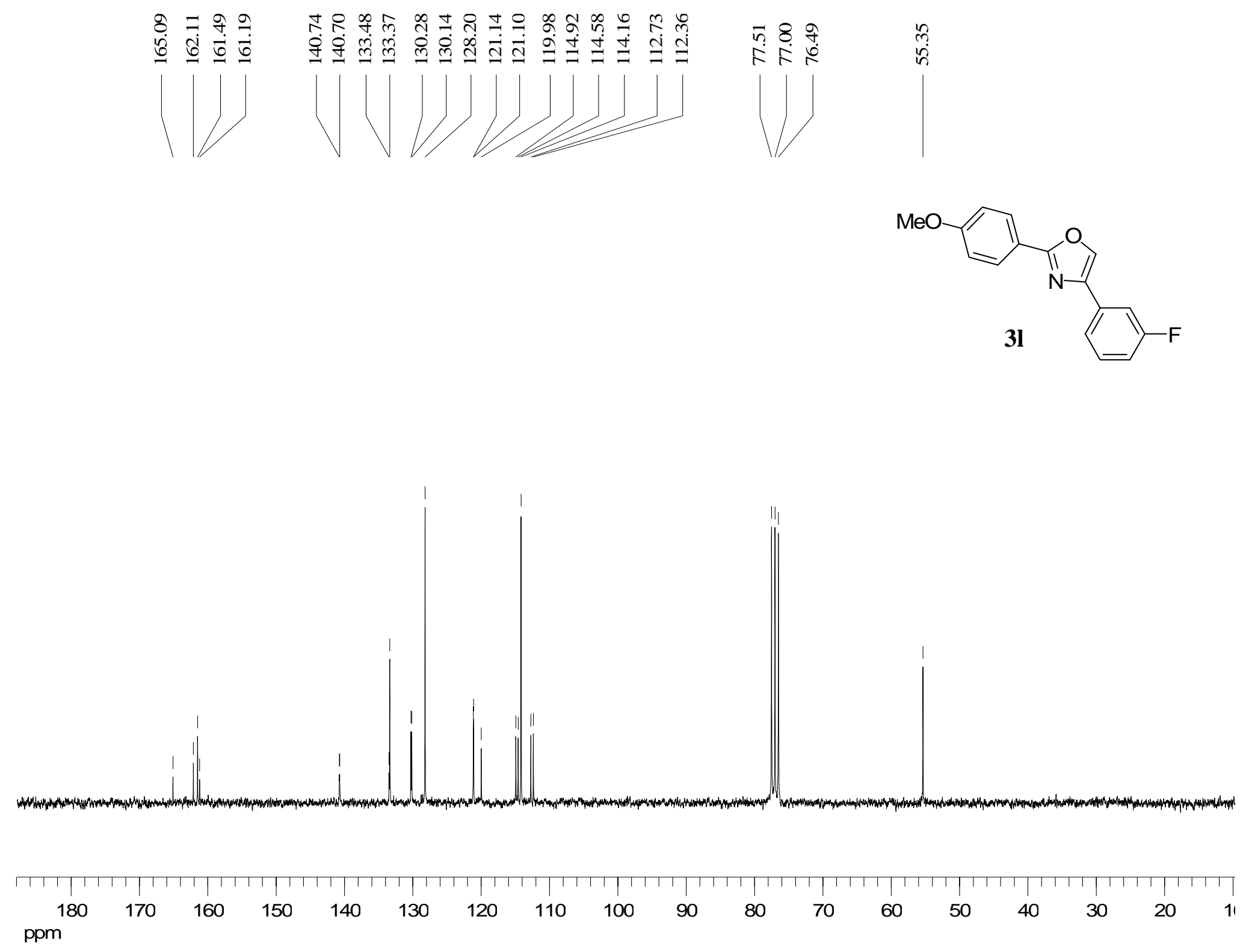


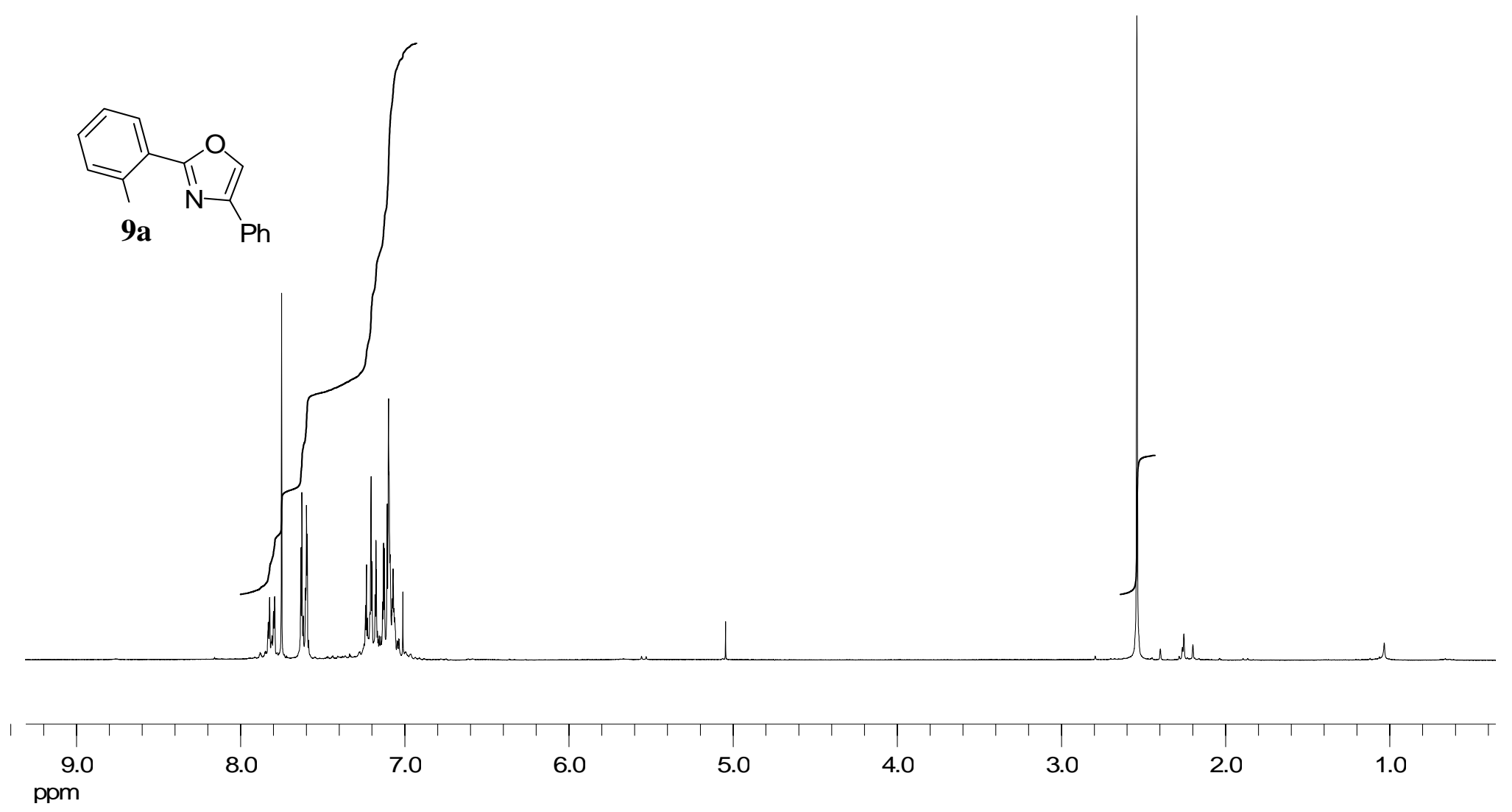

Page 38 

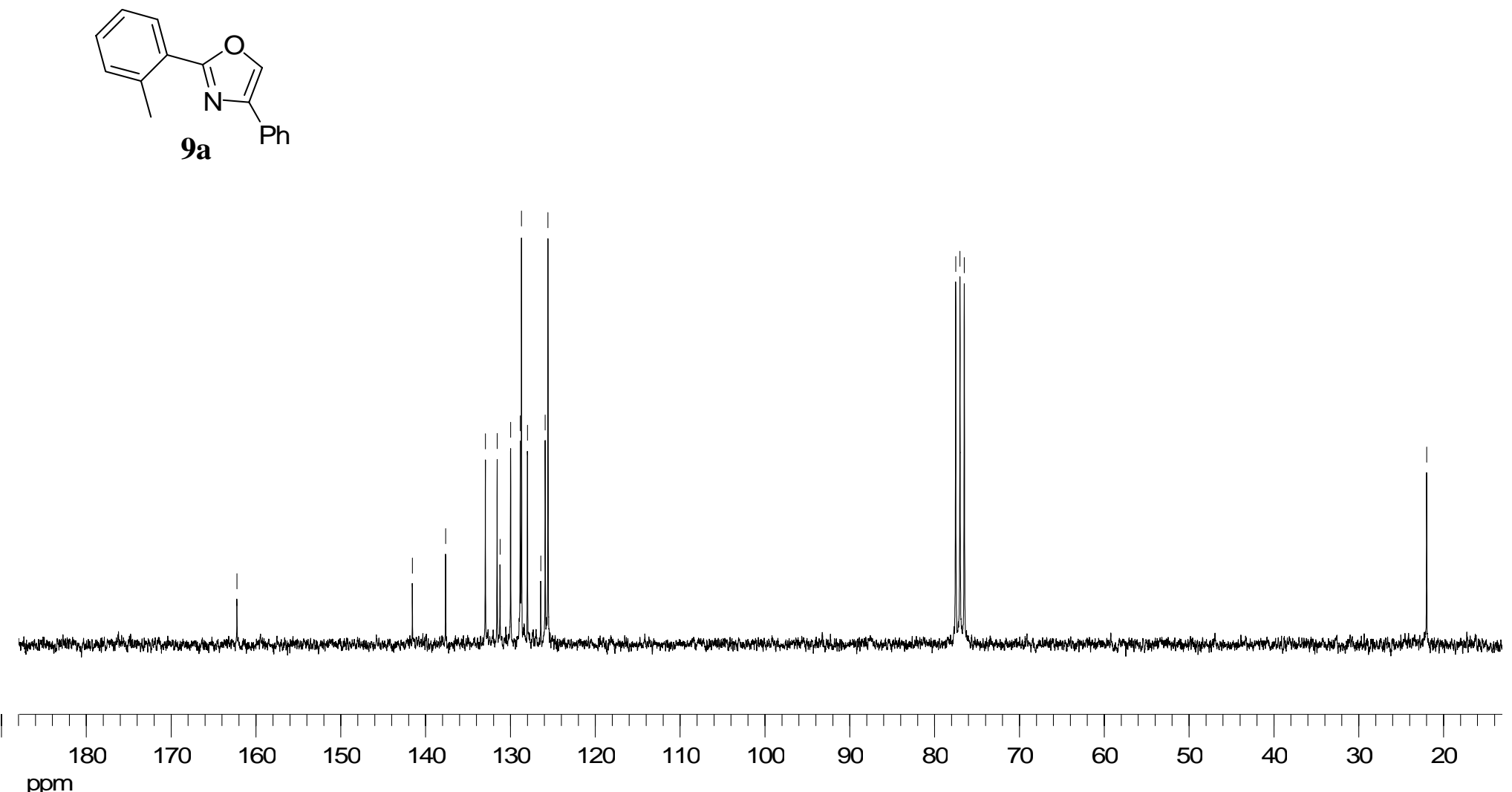


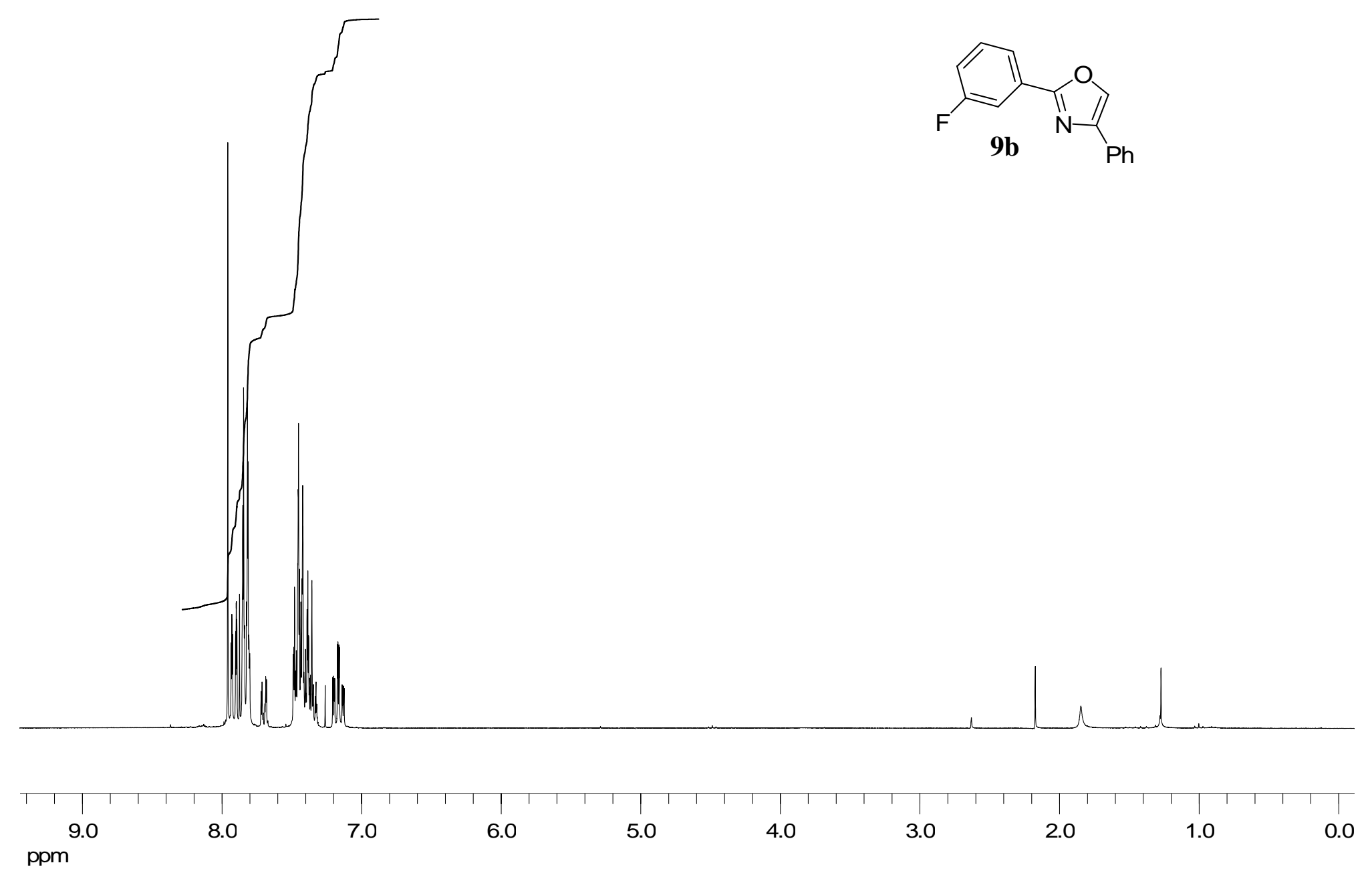

Page 40 


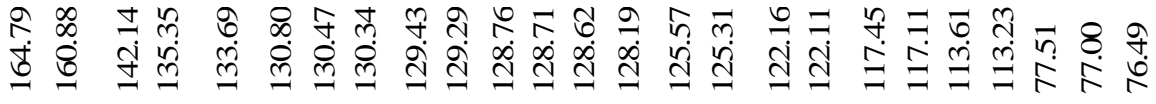
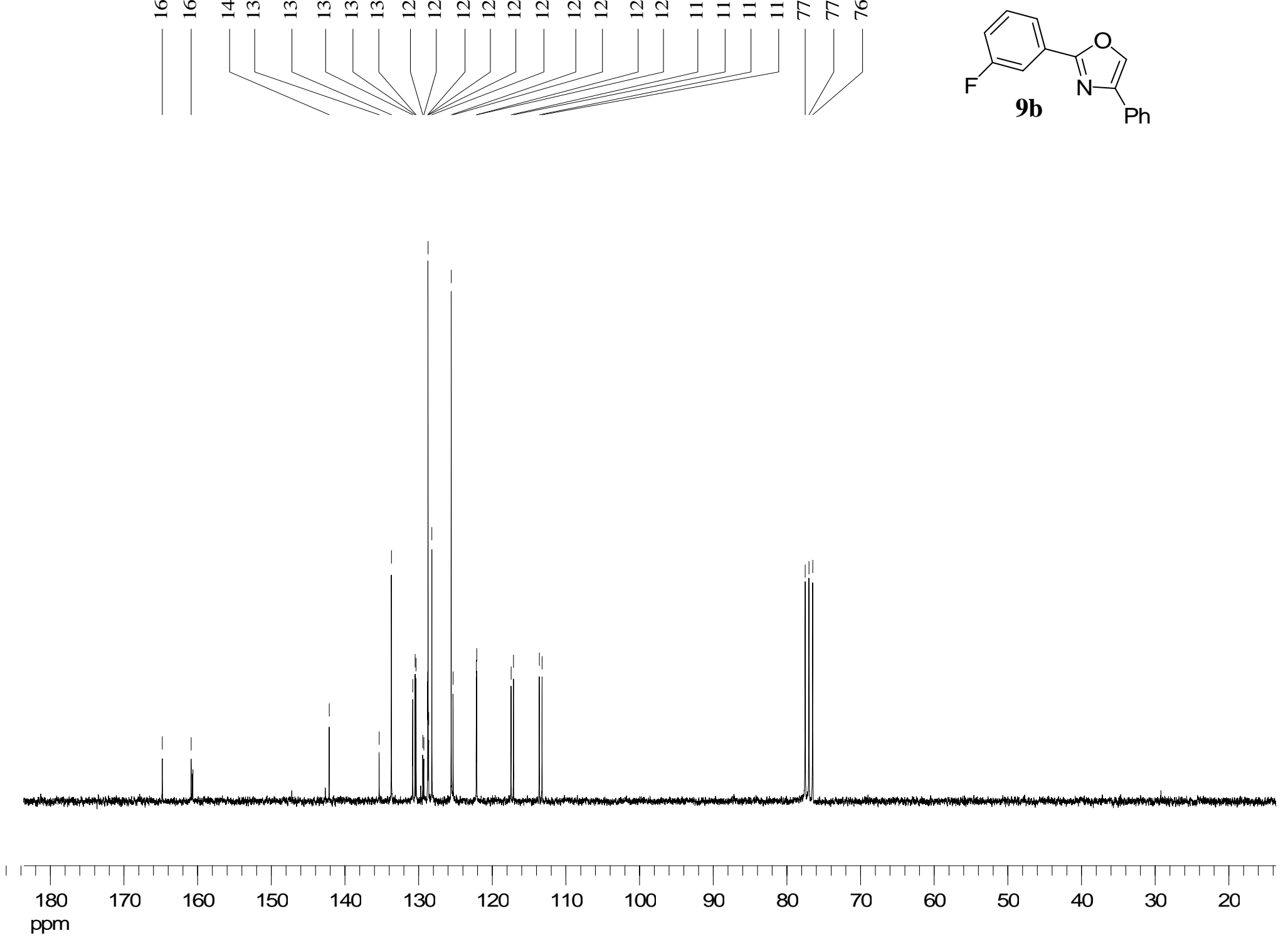

Page 41 


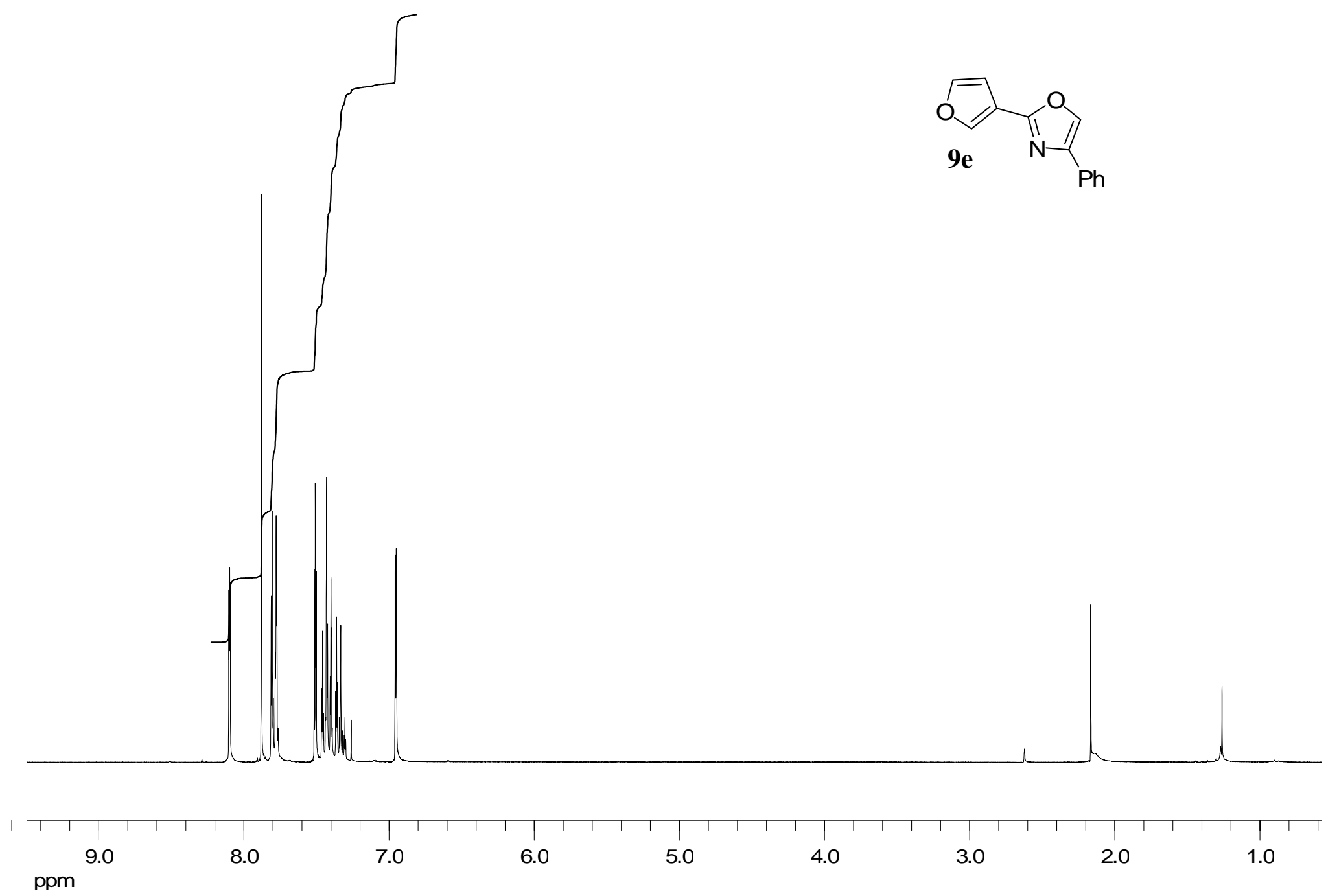

Page 42 

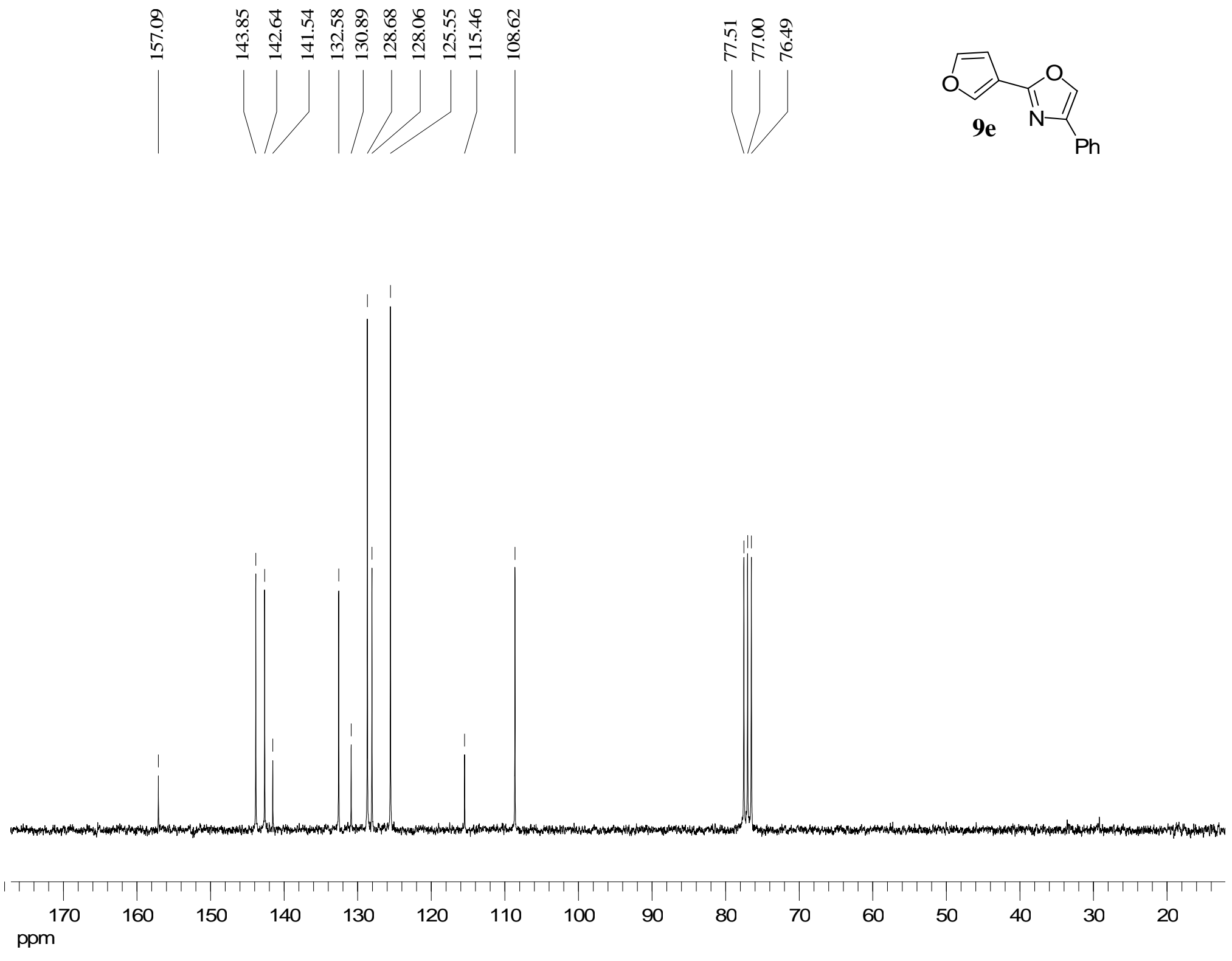

Page 43 


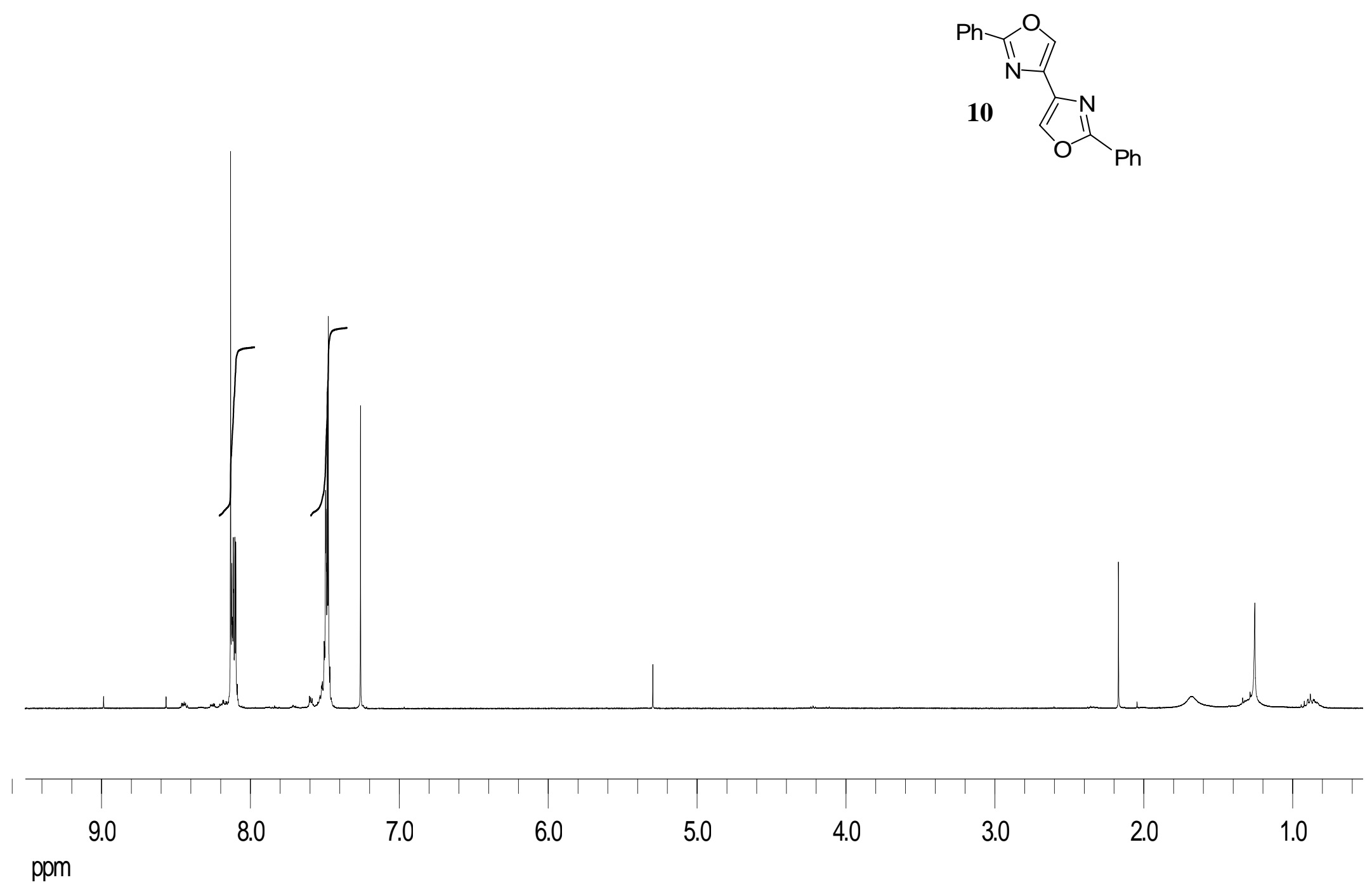

Page 44 


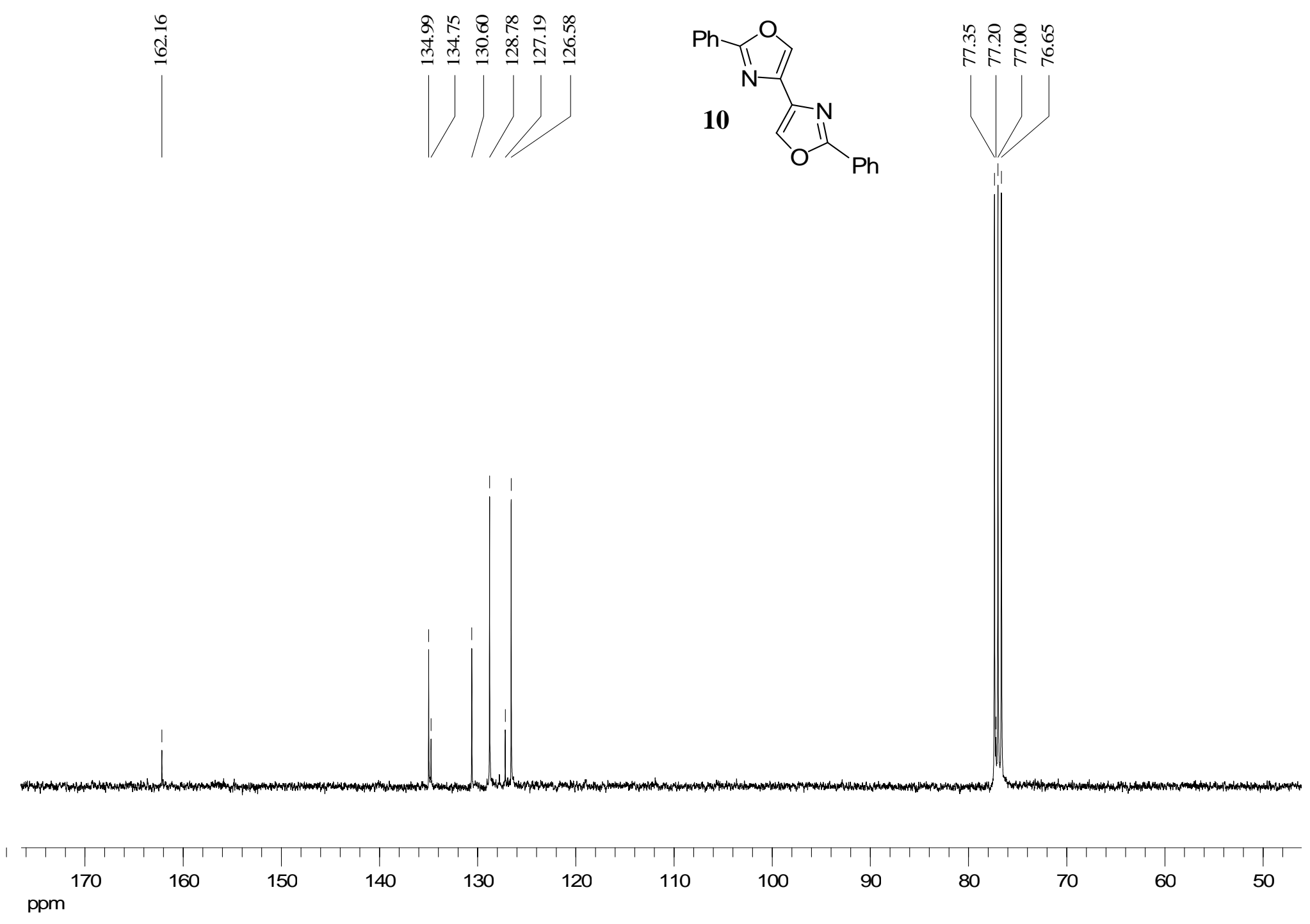

Page 45 


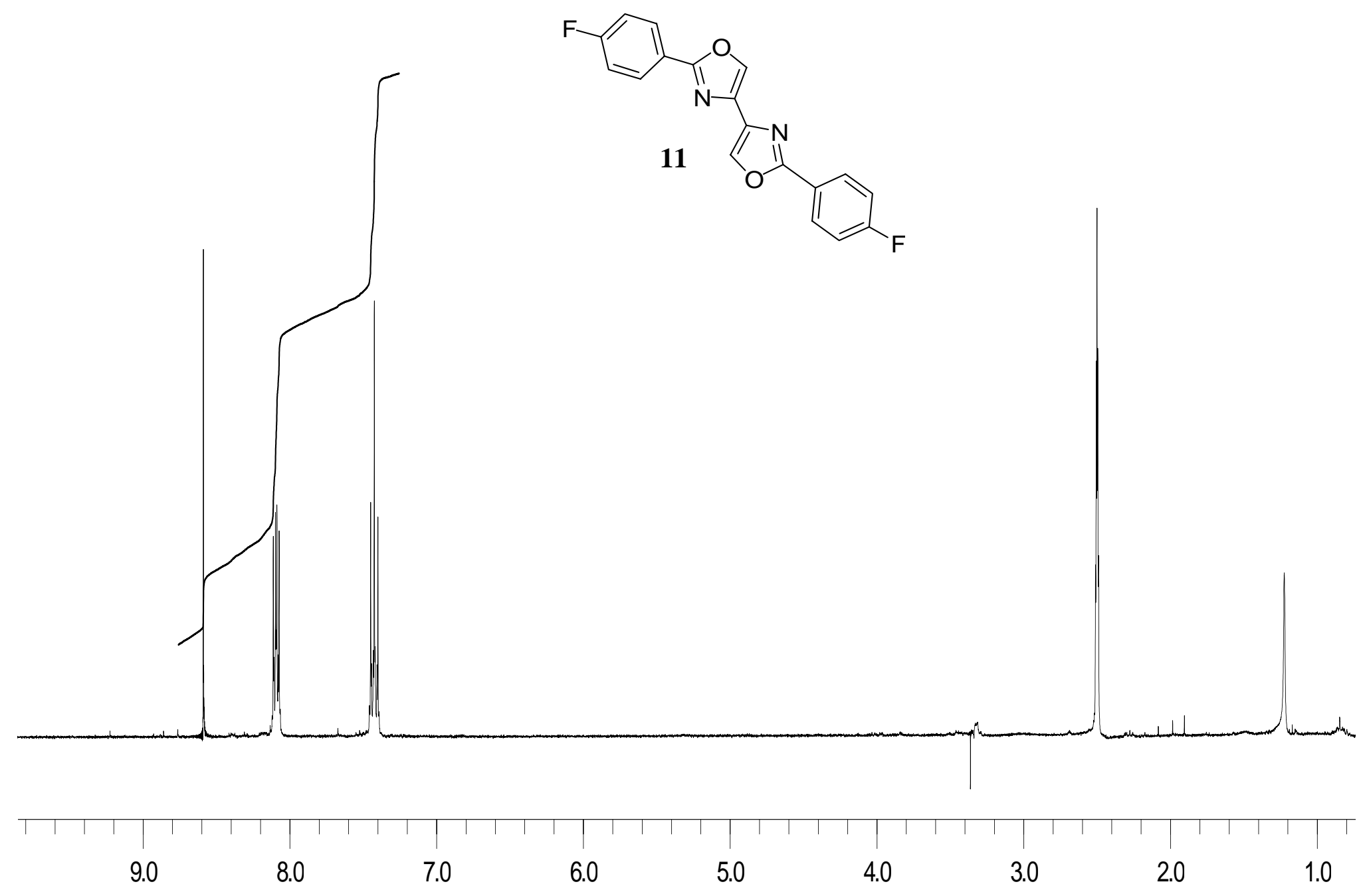

ppm

Page 46 

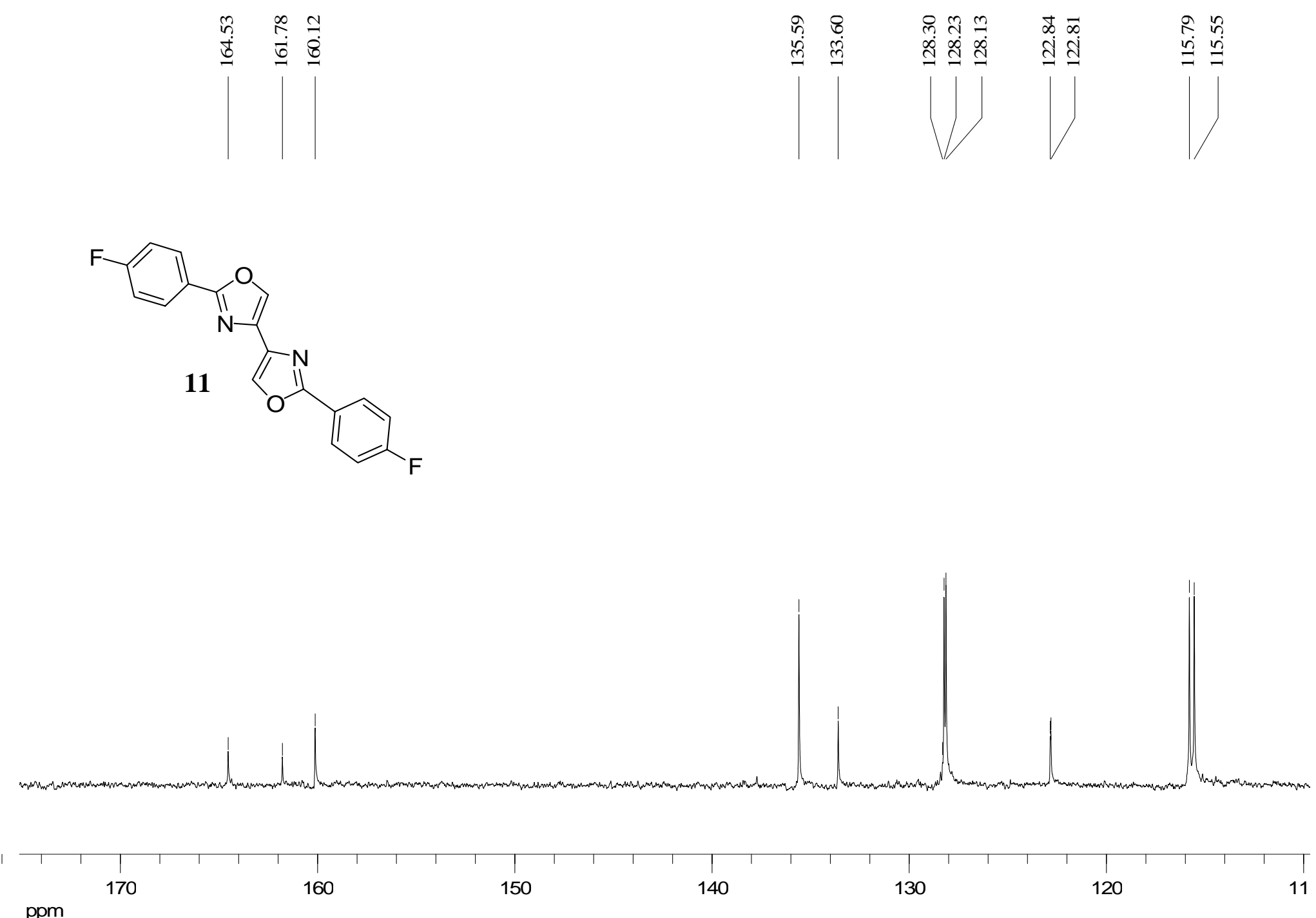


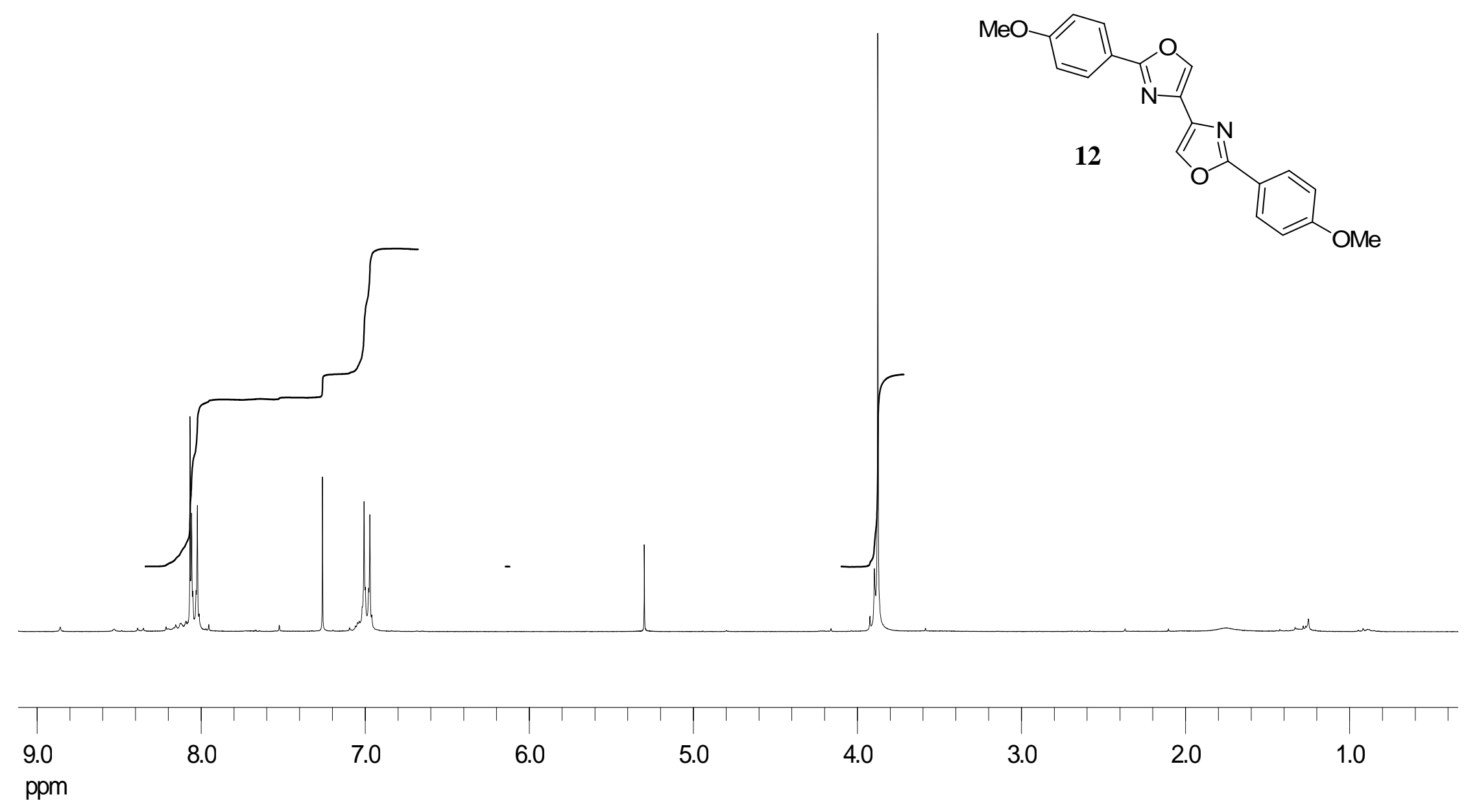

Page 48 


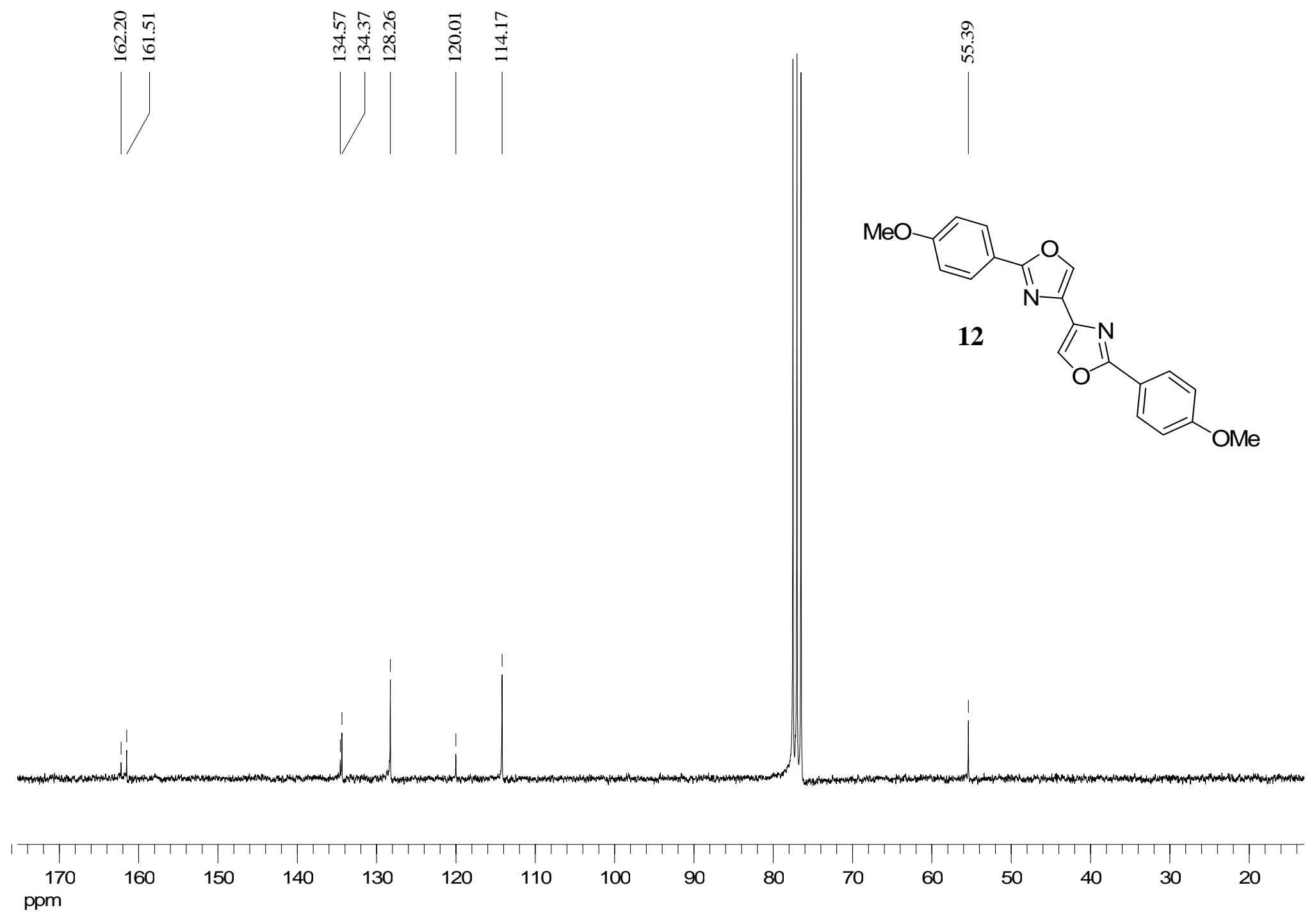

Page 49 


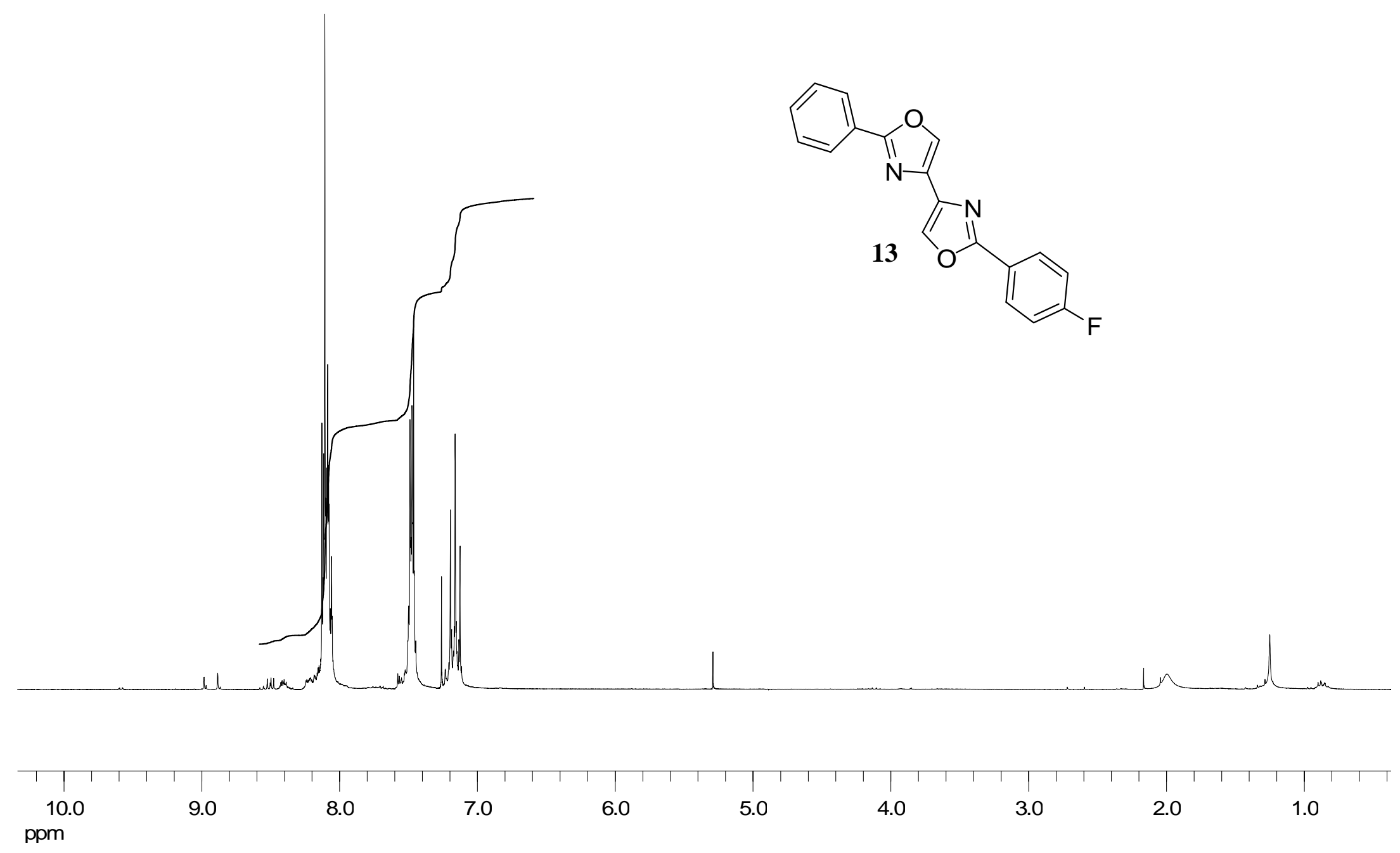




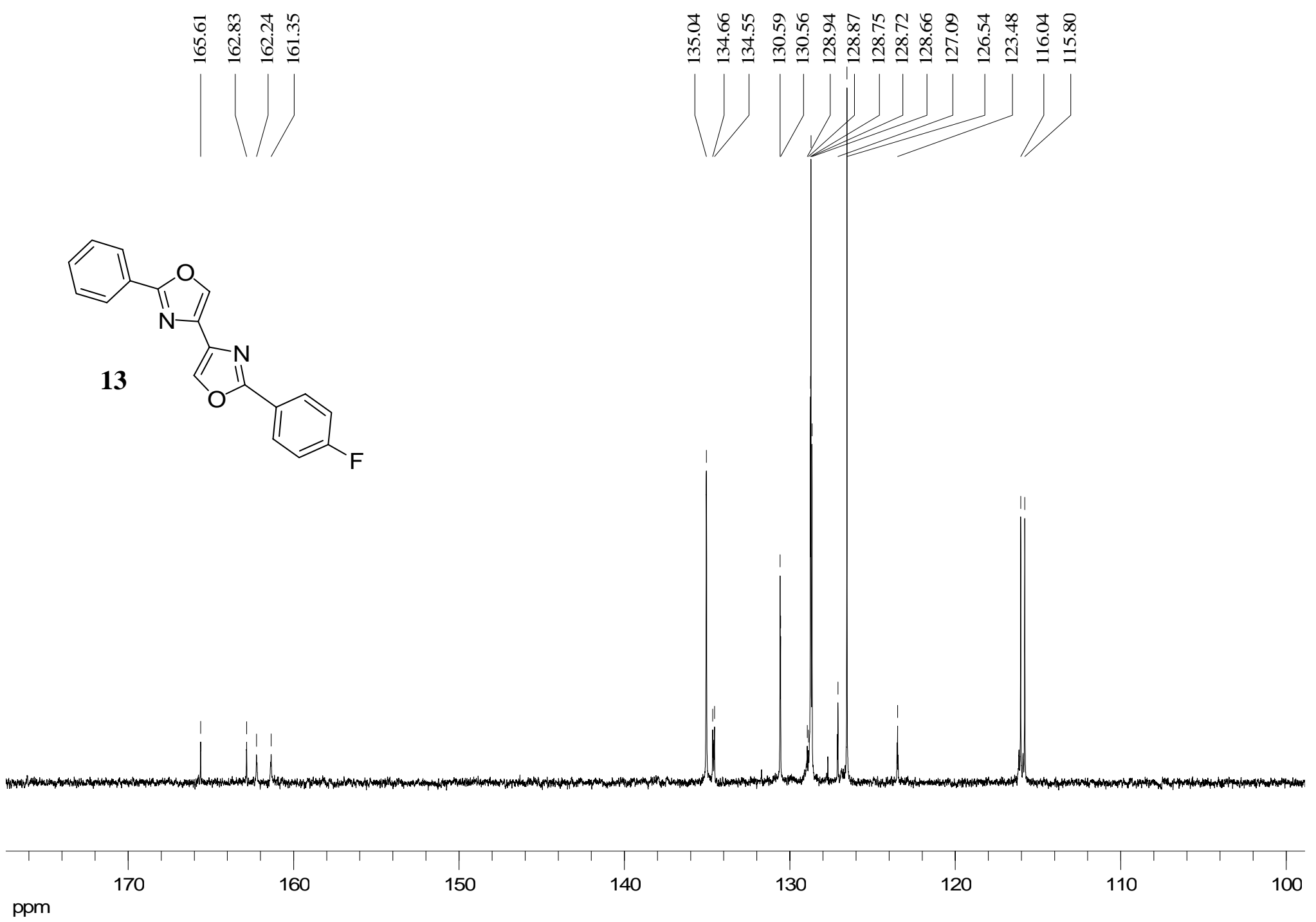

Page 51 


\section{$\underline{\text { References }}$}

${ }^{1}$ Langille, N. F.; Dakin, L. A.; Panek, J. S. Org. Lett. 2002, 4, 2485-2488.

${ }^{2}$ Kelly, T. R.; Lang, F. J. Org. Chem. 1996, 61, 4623-4633.

${ }^{3}$ Maeda, M.; Kojima, M. J. Chem. Soc. Perkin Trans. I 1977, 239-247.

${ }^{4}$ Huang, W.; Pei, J.; Chen, B.; Pei, W.; Ye, X. Tetrahedron, 1996, 52, 10131-10136.

${ }^{5}$ Kauffman, J. M.; Litak, P. T.; Adams, J. K.; Henry, R. A.; Hollins, R. A. J. Heterocycl. Chem. 1992, 29, 1245-1273. 